\title{
DES POUVOIRS
}

\section{DE LA CHAMBRE}

\section{DES REPRÉSENTANS,}

ET

\section{DE L'USAGE QU'ELLE EN A FAIT.}

pAR M. DUCHESNE, DE GRenoble, MEMBRE DE LA CHAMBRE.

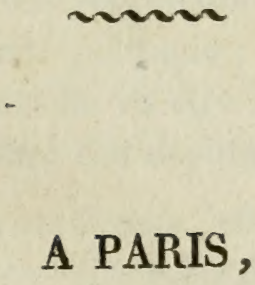

CheZ $\left\{\begin{array}{l}\text { LAURENS-BEAUPRÉ, ITBRAIRE, PALAIS-ROXAL, } \\ \text { GALERIE DE BOIS, } \text { N }^{\circ} 218 . \\ \text { DELAUNAY, LIBRAIRE, PALAIS ROYAL, GALERIE } \\ \text { DE BOIS. }\end{array}\right.$ $1 \overline{815}$ 
Digitized by the Internet Archive in 2010 with funding from University of Ottawa 


\section{DES POUVOIRS}

DE LA CHAMBRE

\section{DES REPRÉSENTANS,}

ET

DE L'USAGE QU'ELLE EN A FAIT.

La chambre des représentans vient de terminer son existence politique; elle a été dissoute par la force, et elle va être remplacée par une nouvelle chambre des députés.

Dans cet état de choses, elle devait s'attendre à être calomniée : des écrivains qui ont tour à tour encensé Napoléon et Louis XVIII, qui ont successivement plaidé la cause du despotisme et de la liberté ; de pareils écrivains auraient démenti leur caractère si, en parlant de la chambre des représentans dispersée et en quelque 
sorte proscrite, ils s'étaient renfermés dans les bornes d'une juste modération.

Aussi la chambre des représentans n'était-elle, selon eux, qu'une réunion d'énergumènes, de factieux, de modernes Brutus, de Napoléonistes déhontés, qui, convoqués par un usurpateur, nommés par une faible minorité (et seulement dans les deux tiers des départemens ) n'avaient jamais eu de titre légitime; qui dans tous les cas auraient perdu ce titre le jour de l'abdication de Napoléon; qui n'auraient jamais eu non plus le droit de reviser notre constitution; qui voulaient sacrifier Paris, l'armée et la France à leurs intérêts, à leurs passions et au triomphe de leurs opinions démagogiques ; qui ont eu l'infamie de voter des remercîmens à Napoléon sur ses défaites; qui dans leurs dernières séances, enfin, n'ont fait entendre que d'affreuses vociférations, n'ont enfanté que des actes de folie.

Sans doute que ces dégoûtantes déclamations ne parviendront pas à empoisonner, aux yeux de la saine partie de la nation, les intentions dont la chambre des représentans était animée. Cependant, comme l'accusation a reçu une grande publicité, comme elle est restée jusqu'icr sans réponse, il peut être utile de rappeler 


\section{(3)}

quelques principes, de rétablir quelques faits qui ont été dissimulés ou dénaturés, et qui jetteront un grand jour, soit sur la légitimité des pouvoirs de cette assemblée, soit sur l'usage qu'elle en a fait.

Le premier reproche qu'on lui adresse, c'est celui d'avoir été nommée à une époque où la France était au pouvoir d'un usurpateur.

Quelques personnes pourraient répondre qu'à cette époque Napoléon élait arrivé sans obstacle jusqu'à Paris; que les troubles du midi étaient - apaisés ; que ceux de la Vendée n'avaient pas commencé ; que de tous les points de la France on faisait parvenir au nouveau gouvernement des adresses de félicitations semblables à celles qui, l'année précédente, avaient accueilli LouisXVIII; qu'ainsi il était permis de se faire illusion sur les droits de Napoléon.

Mais, il faut l'avouer, la soumission apparente des citoyens, les voux par eux consignés dans quelques adresses ne font pas foi de leurs véritables sentimens; et ces sentimens, quels qu'ils pussent être, auraient eu encore besoin d'une sanction plus régulière. Ainsi il est très- 


\section{(4)}

vrai de dire qu'au moment où les colléges électoraux ont été convoqués, Napoléon n'était pas le chef légitime de la nation.

Peut-être mểme pourrait-on aller plus loin ; peut-être pourrait-on soutenir que malgré l'approbation donnée postérieurement par quinze cent mille citoyens à l'acte additionnel aux constitutions de l'empire, les droits de Napoléon étaient toujours problématiques; que quinze cent mille citoyens ne forment pas à beaucoup près en France la majorité des personnss aptes à voter; que d'ailleurs le mode établi pour constater les votes ne laissait pas la faculté d'émettre librement son opinion; que, par conséquent, cet acte additionnel (quoique revêtux d'un plus grand nombre de suffrages que la charte de 1814 , émanée de la toute-puissance de Louis XVIII, et qui n'a jamais pu avoir en: sa faveur qu'une adhésion tacite et présumée) n'était pas encore l'expression vraie de la volonté générale.

Enfin, on ne peut se le dissimuler, la majorité des Français eût-elle librement accepté l'acte additionnel, Napoléon n'en serait pas redevenu, par cela seul, le chef légitime de l'État, s'il est vrai (comme quelques personnes le soutiennent) 
F̧a les droits d'un monarque au trône dépen(ent exclusivement du hasard de la naissance, qu'ils subsistent malgré la volonté contraire de la nation, et qu'ils ne sont même pas présupposiifs d'un contrat régulier intervenu originairement entre le peuple et son chef.

Que faut-il en conclure? que les nominations faites par les colléges électoraux ont été, dès lors, entachées d'un vice radical? Non, sans doute.

Dans un gouvernement représentatif comme celui de la France, il convient de distinguer les élections qui doivent être faites par le peuple ou per ses mandataires, de celles qui appartiennent exclusivement au monarque.

Celles-ci ne sont pas toujours régulières : la raison en est qu'elles peuvent quelquefois émaner d'un chef que la violence, que la force seule ont créé; et que dans ce cas, il y a une véritable usurpation de pouvoirs.

Mais il en est tọut autrement de celles que le peuple s'est réservées : peu importe que le chef de l'état soit légitime ou non; le droit que le peuple avait d'élire est toujours subsistant,-parce que sa qualité originaire n'est point altérée, 
parce qu'au milieu des bouleversemens politiques qui ont ébranlé le trône, il est même plus que jamais une autorité légitime par excellence.

Par conséquent, rien n'est plus clair : la régularité des élections faites par les colléges électoraux, dans ces derniers temps, dépend du seul point de savoir si on y a procédé régulièrement.

Vainement objecterait-on qu'à l'époque où les colléges électoraux ont été convoqués, il existait une chambre des députés dont les pouvoirs n'étaient pas expirés, et que le droit qu'a le peuple d'élire ses représentans, ne s'applique dans aucun état de cause au cas où il est déjà représenté.

La chambre des députés se composait des membres de l'ancien corps législatif qui n'avaient pas été nommés directement par les colléges électoraux, qui avaient été élus pour un laps de temps déjà expiré à l'égard de la plupart d'entre eux, et dont une simple ordonnance du Roi avait prorogé les pouvoirs : on le -demande à tout homme de bonne foi, étaient-ce là de véritables représentans de la nation?

Mais, observe-t-on, les opérations des colléges 


\section{( 7 )}

électoraux seraient encore nulles, en ce sens qu'on y aurait méconnu ou violé toutes les règles précédemment établies; c'est ce qu'il faut examiner.

On prétend qu'aux termes des lois constitutionnelles alors subsistantes, les colléges électoraux ne pouvaient faire de nominations valables lorsque la majorité des membres dont ils se composent n'y avait pas siégé ; qu'en effet le simple bon sens exclut l'idée que la minorité puisse faire la loi à la majorité; que cependant, dans la plupart des colléges électoraux d'arrondissemens et de départemens, c'est une faible minorité qui a nommé.

Le fait d'abord n'est pas exact : à quelques exceptions près, la majorité des électeurs a pris part aux élections. Cette majorité n'a pas toujours formé un nombre de votans considérable; mais c'est que, dans beaucoup de colléges, les décès, les changemens de domicile ou d'autres causes ont opéré des vides qu'on a jusqu’à présent négligé de remplir.

Ensuite, c'est une grande erreur de croire que dans la rigueur des principes, une élection n'est valable qu'autant qu'elle a été faite par la majorité des membres appelés à voter. Cette règle, qui 


\section{(8.)}

doit en effet s'appliquer aux corps délibérans et amovibles dont les opérations ne se terminent pas dans un délai fixe, n’a jamais été faite pour ceux dont les fonctions sont à vie, et dont l'unique mission est de nommer à certains emplois, à tne époque et dans un espace de temps déterminés. Pour ce dernier cas, il suffit que la majorité soit légalement convoquée; car, autrement, il arriverait presque toujours que l'insouciance des uns, que l'absence ou la maladie des autres, enchaîneraient l'action du gouvernement, priveraient le peuple des bienfaits d'une représentation nationale.

Pour en donner quelques exemples, c'est ains que, d'après la constitution de $179^{1}$, on n'exigeail point des assemblées primaires et électorales qu'elles fussent, à aucune époque, en majorité pour faire leurs nominations, et qu'il en était autrement de l'assemblée nationale législative; c'est encore ainsi que, sous l'empire de la constitution de l'an 5, les deux conseils législatifs devaient être en majorité pour délibérer, tandis qu'aucune condition n'était imposée, quant à ce, aux assemblées primaires et électorales.

Et maintenant, pour ne parler que des règles. qui, dèns ces derniers temps, régissaient les 


\section{(9)}

colléges électoraux, on s'est également rrompé quand on a dit que leurs choix, porir être valables, devaient être faits par la majorité.

Cette obligation ne pourait pas résulter, it leur égard, des dispositions de l'article go de la constitution de l'an 8, portant « qu'un corps ) constitué ne peut prendre de délibérations que 》) dans une séance où les deux tiers de ses mem) bres se trouven ( présens ); et il y a plusieuss raisons à en donner.

La première, qu'à celte époque il n'existait ni colléges d’arrondissemens, ni colléges de départemens.

La seconde, qu'un collége électoral n'est pas nn corps constitué, puisqu'il ne délibère pas, puisque sa seule mission est de faire certaines élections.

La troisième el ciernière, que cet article go avait exclusivement en vue le sénat, le corps législatif ct le tribunat d'alors, qui étaient, eux, de véritables corps 'constitués.

Mais ce que la constitution de l'an 8 ne décidait pus, le sénatus-consulte organique des colléges électoraux ne l'a pas mieux fait : on n'y voitnulle part que la majorité des membres d'un collége 
doive concourir aux élections; c'est donc qu'aux termes de ce sénatus-consulte, comme aux termes des constitutions de 1791 et de l'an 5, les élections d'un collége sont valables, quel que soit le nombre des membres présens.

Cette observation devrait suffire : cependant il n'est pas inutile de faire remarquer qu'aucun sénatus-consulte, qu'aucune loi postérieure n'a établi sur cela des règles différentes de celles consignées dans le sénatus-consulte du 16 thermidor an 10 : dès lors, comment concevoir qu'on ait pu sérieusement arguer de nullité les élections faites dans les colléges électoraux où la majorité des membres du tableau n'a pas siégé?

Le droit commun est très-certainement que, pour des élections de ce genre, il suffit de la majorité des membres présens : par conséquent, dans cette circonstance, il ne faudrait rien moins qu'une dérogation expresse aux anciens principes; or, on ne saurait trop le répéter, elle n'existe ni dans la constitution de l'an 8, ni dans les lois, ni dans les sénatus-consultes; et cependant elle ne saurait résulter que de celle espèce d'actes.

Peu importe en effet qu'un décret impérial postérieur ait décidé que les colléges électoraux 


\section{(11)}

ne pourraient clore leur scrutin que lorsque la majorité des membres du tableau aurait voté : un pareil décret n'est pas une loi, n'est pas un sénatus-consulte; il n'a donc jamais pu être obligatoire pour les colléges électoraux, et encore moins pour des colléges électoraux réunis à une époque où l'autorité de qui ce décret est émané n'avait réellement en sa faveur aucun caractère de légitimité.

Suppose-t-on le contraire? soutient-on que depuis le jour de son arrivée à Paris, et avant même la cérémonie du Champ de Mai, Napoléon a été de fait et de droit le chef suprême de l'État, et qu'en cette qualité il a pu exercer quelque juridiction sur les colléges électoraux?

On accordera tout au moins alors qu'il a été autorisé à rapporter son propre décret, ou à en ajourner l'exécution : or , c'est ce qu'il a fait, puisque l'instruction ministérielle adressée dans le mois de mai dernier aux colléges électoraux leur enjoignait expressément, malgré toutes dispositions contraires, de clore leur scrutin quel que fût le nombre des votans.

Ainsi, dans aucun état de cause, ce décret impérial ne pouvait s'appliquer aux dernières élections faites par les colléges électoraux; ainsi ces 
élections ne penvent être querellées sur le fondement que, daus quelques colléges, les membres présens n'auraicut pas formé la majorité de ceux inscrits au tableau.

On se retranche à la vérité derrière une autre irrégularité; on prétend que le tiexs des départemens s'est refusé à nommer des représentans: d'où la conséquence que la chambre n'était pas complette.

Le fait fût-il vrai, la critique n'aurait aucune espèce de fondement; car un des principes les plus constans en pareille matière, c'est celui qu'une assemblée délibérante est régulièrement formée, dès l'instant que la majorité des membres dont elle doit se composer se troure réunie.

Mais celle étrange assertion prouve à elle seule toute la mauvaise foi des reproches adressés à la chambre des représentans : il est faux qu'aucun collége d'arrondissenent ou de département ait négligé de faire les élections : quelques uns seulement étaient en relard pour l'enroi de leurs procès-verbaux, et dès les quinze premiers jours de la session, tout à cet égard a été régularisé. 


\section{( 13$)$}

Jusqu'ici donc, rien de plus frivole que les diverses objections faites contre les élections des . membres de la chambre des représentans; et il sera facile de s'en convaincre, on n'a pas été plus heureux dans les prétendus empiétemens de pouvoirs qu'on lui a reprochés.

On a dit qu'elle avait été créée par Napoléon, et qu'elle avait dû finir avec lui; que, depuis l'abdication de Napoléon, par conséquent, tout ce qu'elle avait fait était illégal.

Passons sur le danger qu'il y aurait eu à faire dans ce moment l'application d'un pareil principe : mais ou a-t-on pris que la validité des élections faites directement par la nation, et cn vertu d'un droit antérieurement acquis, était subordonnée à un événement comme celui de l'abdication de Napoléon? Une élection régulièrement faite ne peut être annulée qu'autant que l'autorité qui a nommé n'était pas légitime : or, très-certainement une nation dont le gouvernement est représentatif, est autorisée à se nommer des représentans, quel que soit le chef qui tient les rênes de l'Etat.

Qu'a-t-on encore reproché à la chambre des 


\section{(14)}

représentans? de s'être arrogé le droit de reviser nos constitutions, conjointement avec la chambre des pairs et le pouvoir exécutif.

Or, sans insister sur d'autres considérations tout aussi puissantes, il est étrange que ce droit lui soit contesté, par des personnes qui supposent, qu'une des trois branches de la puissance législative a pu, non pas seulement réviser notre constitution, mais la faire; il est encore plus étrange que ce droit lui soit contesté, quand on admet d'un autre côté que trois corps distincts doivent concourir à la formation de la loi. C'est donner à entendre qu'une constitution est moins qu'une loi, et que le respect dont on l'entoure est une ridicule superstition.

Il faut donc être de bonne foi, et avouer que la chambre des représentans a été régulièrement nommée, qu'elle a pu siéger après même l'abdication de Napoléon ; qu'enfin elle a pu s'occuper de la révision de la constitution.

Reste, à la vérité, la question de savoir si le mandat qu'elle avait reçu, elle l'a bien et fidèlement rempli : mais si c'est ici que les reproches s'accumulent, c'est également ici que 
les réponses sont plus décisives's el plis victorieuses.

On accuse la chambre des représentan: d'aroir constamment rampé anx pieds de Napoléon !.... On ne se rappelle donc pas que son chois pour un président, s'est porté sur un homme bien connu par lia noble résistance qu'il arait constamment opposée au despotisme de l'Enipercur! On oublie donc que daus son adresse à Nipleléon, elle lui a tenu un langige dont ses oreilles ont élé effarouchées? On oublie donc qu'elle a repoussé avec une sorte d'inclignation lia proposition qui lui élait faite, de conférer a Napoléon le surnom de sauveur du peuple, et qu'elle aurait pu. cependant s'antoriser de l'exemple que la chambre des députés lui avait donné un an auparavant! On ne lui sait donc aucun gré de celte al, dication qu'elle a provoquée et obtenue à une époque où tant d'intérêts et de passions s'y opposaient!

On prétend qu'elle a vorih yégner par la terreur !.... Et cependant, malģxé l'ordomiance du Roi, qui condamnait Napoléon et ses adhérens à la peine capitale, eile n'a point voulu user de représailles; elle n’a pris conire les partisans de. Louis XVIII que des mesures de surveillance dont. 


\section{( 16 )}

elle s'est même efforcée d'adoucir la sévérité ! et cependant elle a décidé que l'abolition de la confiscation serait l'objet d'un des articles de la constitution! et cependant enfin, à la simple lecture d'un projet qui mettait les Vendéens hors de la loi, tous ses membres se sont écriés qu'ils n'étaient pas des assassins!

On lui fait un crime des remercîmens qu'elle a votés à Napoléon après son abdication !.... Eh ! ne sait-on pas que ces remercîmens ne s'adressaient ni à l'Empereur ni au Général; qu'ils avaient pour unique molif d'empêcher une rétractation qui aurait été suivie des plus grands malheurs? Ne sait-on pas que la bienséance commande souvent des démarches que l'austère raison désavoue?

On aurait voulu du moins qu'après cette abdication, la chambre se prononçât de suite en faveur de Louis XVIII !... qu'en serait-il résulté? que Napoléon aurait regardé son abdication comme nulle, qu'il serait allé se replacer à la tête des troupes, et que le sang aurait coulé de nouveau par torrens. Au surplus, la chambre était liée par un serment qu'elle avait prêté avec tous les fonctionnaires de l'état, et dont l'abdica1ion de Napoléon ne l'avait nullement relevée : 


\section{(17)}

aux termes de ce serment (et tant que la nation n'avait pas manifesté un vœu contraire d'une manière anthentique ) elle ne pouvait reconnaître pour chef que le fils de Napoléon : elle n'avait qu'un parti à prendre pour tout concilier; c'était celui de consulter la nation sur un changement de dynastie, en même temps qu'elle la consulterait sur la constitution dont elle discutait les bases. Or, c'est ce qu'elle aurail fait, si les événemens le lui avaient permis; car plusieurs des articles de cette conslitution indiquaient clairement la possibilité clu retour de Louis XVIII.

Mais s'occuper d'une constitution, c'était mettre des conditions à ce retour, et dans le nombre il y en avait d'inadmissibles; telles étaient l'abolition de la noblesse, le licenciement des gardes du corps, et l'obligation d'adopter la cocarde tricolore... Sur l'un et sur l'autre point l'impartiale histoire prononcera : elle dira si la chambre des représentans a eu tort de demander des concessions réclamées à grands cris par la classe la plus nombreuse de la société, et sur lesquelles les personnes qui approchent Louis XVIII de plus près, se sont rendues auprès de lui les intcrprètes de l'opinion publique. Elle dira, enfin, si de la part de la chambre des représentans, ce fut une. 


\section{2}

tẻmérité trop grande de conisacrer le principe que le grand ourre d'une constitution ne doit pas émaner exclusivement du monarque, et de signaler les imperfections d'une charte à laquelle on croit devoir faire dès à présent d'importantes modificalions.

On se: plaint de ce que la chambre des représentans, a cherché ì prolonger dans son intérêt une litte devenue tout-ì-fait inégale ?.... Ce reprocle est an moins injuste : dès le lendemain do Jabdication de Napoléon, clle a chargé le gouvernement provisoire de traitcr avec les puissinces étrangères, et elle n’a cessé d'émottre le vœu que la paix vint meltre promptement un terme aux malheurs de la France. A la vérité elle aurait désiré que la capilale pùt ćchapper au fléan d'une occupation militaire, et imul qu'elle en a entrevu la possibilité, elle a cru devoir färe un appel au combige de l'amée el des citoyens. Avail-elle tort?

On s'étonne qu'an pius fort de la crise toutes ses délibérations n’aient pas été également calmes el mijestucuses: on a parlé de ces sénateurs romains qui se laissèrent massacrer sur leurs chaises curules, où ils avaient conservé toute la gravité de leur caractère !..... Mais après la bataille de 


\section{(ig)}

Cannes, les Romains ne désespérèrent pas du salut de la patrie, et leur attitude resta constamment noble et ferme; chez nous, au contraire, une bataille perdue plonge dans le plus profond abattement, ou met toutes les passions en mouveınent. Français, n’exigez pas de vos représentans des vertus que vous n'avez pas vous-mêmes, ct qu'aucune de vos assemblées délibérantes n'a pratiquées.

Ce sont des acles de déraison et de folie qui ont terminé la session de la chambre des représentans !.... Quniqu'en puisse dire la malignité et I'esprit de parti, la chambre des représentans n’a ricn à redouter du jugement qu'on portera sur les derniers actes émanés d'clle; le projet de consitution qu'elle a disculé dans ses trois dernières séances, valait peut-être la cluarte de 1811; il n'est, ul l'oeurre de la démence, ni le Code de l'anairchie.

On ajoute que la chambre des représcutans s'est mise en révolte ouverte contre Louis XVIII en ne se séparant pas d'clle-même au moment où le gouvernement provisoire lui a annoncé qu’il cessail ses fonctions !..... Soyons justes : Ja chambre pouvait-elle oublier qu'elle tenait ses pouvoirs du peuple, et ane de parcils pourvirs snrvivent 


\section{(20)}

au chef sous l'empire desquels ils ont été conférés? Pouvait-elle oublier qu'aux termes de la charte de 1814 , comme aux termes de l'acte additionnel, sa dissolution, pour être légale, devait lui êlre notifiée par la puissance exécutive? et quand l'antorité compétente gardait le silence, pouvail-elle prendre une honteuse initiative, et prononcer contre elle-même une peine qu'elle ne croyait pas avoir méritée?

S'agit-il maintenant des membres de la chambre qui ont signé la protestation du lendemain? Iis se sont bornés à constater que l'entrée de la salle leur avait été refusée, et qu'une force supérieure les obligeait à se séparer. Ils n'ont point cherché à retarder la marche des événemens, à comprimer l'expression des sentimens qui paraissaient se manifester, et à retenir une autorité qui pouvait flatter leur amour-propre : ils n'ont eu qu'un but, celui de prouver à leurs commettans qu'ils avaient été, jusqu'au dernier moment, fidèles à leur mandat : ce devoir une fois rempli, ils se sont unis d'intention à tous les bons citoyens, et ils font, comme eux, des vœux bien sincères pour que des jours plus doux lui-sent enfin sur notre malheureuse patrie, pour 


\section{( 21 )}

que la concorde nous ramène à sa suite l'abondance et la liberté.

Que reste-t-il donc de tant et tant de reproches adressés à la chambre des représentans? Nonımée légalement par des colléges électoraux convoqués d'une manière régulière, tant que Napoléon a tenu les rênes de l'État, elle a combattu le despotisme et repoussé l'adulation : après avoir provoqué et ubtenu son abdication, et dans des circonstances plus que délicates, elle a cherché à tenir un juste milieu entre les exagérations de chaque parti; et si la guerre civile n'est pas venu mêler ses horreurs à celles d'une invasion étrangère ; c'est peut-être à elle qu'on en est redeváble.

Elle a pu se tromper quelquefois : mais ses intentions étaient pures; mais elle marchait entre deux écueils également redoutables; mais enfin, avec de plus grands talens et des intentions non moins droites, l'assemblée constituante s'est aussi trompée.

Un jour viendrạ où l'on pèsera, dans le calme des passions, les prétendus torts de la chambre des représentans, où on leur opposera les services non contestés qu'elle a rendus à la chose publique. 


\section{(22)}

Alors peut-être on dira que, maîtrisée par les événemens, elle n'a pas fait tout ce qu'on attendait d'elle, mais qu'il était difficile de faire mieux.

DE L'MPRIMERIE DE J. GRATIOT. 




\title{
NOTE SECRETE
}

\author{
EXPOSANT
}

\section{LES PRETTEXTES ET LE BUT \\ -}

\section{DERNIERE CONSPIRATION.}

\section{A PARTS,}

Cliez FOULON ex C., libraires, rue des Francs-Bourgeois-SaintMichel, $n 0^{\circ} 5 ;$ DELAUNAY Lx PÉLICIER, Palais-Royal; Ex CXMERY, zue Mazarine; $\mathbf{n}^{\circ} 3 \%$. 



\section{AVERTISSEMENT DE L'EDITEUR,}

manom

L.

Note secrìte à laquelle on croit utile de donuer une grande publicité pour faire évanouir les insinuations perfides et les calomnies dangereuses qu'elle renferme contre le gouvernement du Roi et contre la nation, a dû être sounise, il y a trois mois, à quelques anbassadeurs des puissances alliées par des négociateurs anonymes, sans mission et sans caracière, qui se prétendent les organes d'un parti.

Il a toujours existé en France, depuis la resiauration, un parti qui a rejeté la Charte, ou qui la présentait comne une simple carte d'entrée, comme une concession nécessaire, mais momentazée. Ce parti agissait dans l'ombre. Il calomniait, dans des notes clandestines, adressées aux cabinets étrangers, le monarque et la nation. Il tendait à exciter, dans ces cabinets, une plus grande disposition à la défiance contre le gouvernement de la France, et à faire prolonger les souffrances de l'occupation armée. Mais on manquait d'une pièce positive qu'on pût regarder comme le manifeste et la profession de foi de ce parti. Cette pièce est tombée entre nos mains : elle nous vient d'une source digne de foi; 


\section{(4)}

elle porte d'ailleurs avec elle, par la manière dont elle est rédigée, un caractère d'authenticité.

Du reste, nous ne nous permettrons ni d'en désiguer ni d'en soupçonner les auteurs. Qu'on ignore à jamais, s'il est possible, les noms de ces indignes Français! mais que leurs calomnies, qui pourraient être accueillies au loin si elles n'étaient promptement réfutces, subissent la juste puuition de la publicité! Le bon sens national eu fera justice.

Il suffit que cette pièce ait existé, qu'elle ait une destination connue, pour qu'il soit convenable et utile de la produire an grand jour, pour faire apprécier aux bons esprits et aux cours français linconvenance et le danger de ces machinations ténébreuses, dont le but est d'offrir toujours la France conme un épouvantail à l'Europe, et de nourrir les prérentions et les haines nationales, qu'il est si important de détruire.

Ce honteux appel aux élrangers, pour fitire changer par lcur influence le systène du gowernemont, scra désavoué par ceux-mêmes qu’un moment de vertige a pu égarer au point de leur suggérer de pareils blasphênues. Car cctle pièce réunil les trois caractères d'un acte de souveraineté, d'un manifeste, et d'un plan de conspiration, en un mot, d'un crime de trahison envers la nation et le Roi. 


\section{NOTE SECRETE}

EXPOSANT

\section{LES PRÉTEXTES ET LE BUT}

D T. LA

DERNIÉRE CONSPIRATION.

\section{A PERÇU \\ DE LA SITUATION DE LA FRANCE.}

AU MOIS DE MARS 1818 .

A Ux époques dn mois d'août 1816 , et au mois d'août 1817 , nous nous sommes efforcés, dins les notes que nous avons fait parvenir aux quatre cours alliées, de montrer par quelle série d'événemens le gouvernement de France s'était éloigné peu-à-peu de la ligne qui pouvait assurer l'établissement du Roi; et nous avons cherché à faire voir comment, en ne prenant aucun des moyens nécrssaires pour établir la monarchie, on préparait le triomphe de la révolution.

Ces opinions paraissaient alors partiales, et elle trouvaient des contradictcurs dans ceux qui n'avaient pas assez observé la France, la marche des espris, et la nature du gouvernement qu'elle essayait. Anjourd'bui le mal est tel, les intentions révolutionnaires sont tellement à découvert, et si pulitiguement avouécs, que les esprits les plus obstiués ont été obligés de se rendre à l'évidence, 


\section{(6)}

et qu'il n'en est plus un seul qui se refuse à déclarer que le Roi est placé sans appui au nilieu du torrent de la révolution.

En effet, la révolution occupe tout depuis lo oalinet du Poi, qui en est devenu le foyer, jusqu'aux dernières classes de la nation qu'elle agite partout avec, violence.

Les principes destructeurs de notre monarchie sont professés à la tribune par des ministres du Roi (a).

Des écrits audacieux sapent tous les fondemens de l'ordre social $(b)$, et les lois répressives ne font obstacle qu'aux écrivains qui soutiennent la monarchie et la légitimité; les jugemens des tribunaux, ce qu'il y a de plus sacré dans les institutions humaines, sont livrés aux diatribes les plus virulentes $(c)$; tous les liens de l'état social sont relâchés; le gouvernement ne paraît marcher que par l'impulsion d'un pouvoir qui n'esiste plus el par la présence des forces étrangères; enfin, tout se prépare à chasser la maison de Bourbon et à faire la guerre à l'Europe.

Un accord aussi parfait pour juger le mal, semJerait annoncer nécessairement une disposition unanime sur les remèdes qu'on pourrait encore y

(a) Voyez le discours du ministre de la police sur la Iiberté de la presse, et celui du ministre de la guerre sur la loi du recrutement.

(b) Comme la Minerve, le Recueil des Pièces historiques, les Lettres Normandes, etc., etc.

(c) Voyez le Mémoire du colonel Fabvier sur les événemens de Lyon, les Lettres de Benjamin Constant dans I'affaire de Wilfrid-Regnault, et quelques articles des Quvrages cilés dans la note précélente $b_{\text {. }}$. 


\section{( 7 )}

apporter; mais il n'en est pas ainsi : les esprits; diversement préoccupés par des antécédens qu'on ne veut pas oublier, parce que personne ne veut avouer qu'il s'est trompé, jettent une grande diversité sur la manière d'envisager les moyens qu'on aurait de réparer le mal qui à été fait, et de se préserver de celui qui menace l'Europe.

Cependant, pour traiter celte importante question, cette question qui renferme le salut ou la perte de tous, il suffit, sans récriminer sur le passé, de partir du point actuel, de ce sentiment, de cet aveu unanime : "La position et la marche 》) actuelle du gouvernement de la France con2) duisent au triomphe certain et prochain de la 2) révolution. ))

Dans cet état de choses, il n'y a pour l'Europe que deux hypothèses: ou l'on abandonnerait la France à toutes les irruptions du volcan, en cherchant à s'en préserver au-dehors; ou l'on penserait à sauver la France de toutes ses fureurs.

En examinant la prenière, on conçoit, en effet, que les cours alliées, qui ont deux fois soulevé l'Europe, versé le sang, prodigué les trésors de leurs sujets pour terrasser cctte révolution; on conçoit que les souverains, qui l'avaient deux fois vaincue, et qui croyaient dans leur sagresse et dans la pureté de leurs intentions avoir tracé la marche politique qui devait à jamais prévenir son retour, soient fatigués de la voir rentître sans cesse et produire de nouvelles têtes à mesure qu'or les a abatturs.

Mais dans ce terrible combat, rien n'est fait quand il reste quelque chose à faire, ct nous avons assez prouvé, dans la note du mois d'août 1817 , combien serait folle l'esperance de se rendre maître 
de l'uncendie grand on lui anrait donné la France entière pour aliment. Et comment espérerati-on de s'en préserver quand il a grandi et acquis des forces dans le temps oil les troupes et les conseils de l'Europe occupaient le territoire de la France, et dirigeait la conduite politique de son gouvernement? entin, qui oscraut penser qu'on saura s'en défendre quand elle attaquera avec tontes ses forces et sa violence, lursqu'on n'a pas su l'étouffer à sa renaissance $(d)$ ?

Loccupation réclle du territoire était justifiée

(d) Trtrait de la note du mois d'aont 1817 . Et quand, sous la protection des souverains alliés, la révolution sera devenue maitresse en France, quelle sera la pasition de cess sonverain alliés eux-mèmes, et quelle conduite tiendront-ils? Doivent-ils dire, comme en $179^{3}$, que peu leur importe les monvemens de la France, quils sauront bien s'en garantir? Ils savent bien que la révolution irait encore les chercher, et qu'elle jetterait sur eux à-la-fois ses opinions et ses armées. Lt, en effet, quel est le chef révolntionnaire qui pourrait essayer de gouverner la France sans le prestige des conquêtes, sans l'aliment de la guerre, et sans donner l'Europe à dévorer à l'avidité et aut fanatisme ambitieux de ses prosélytes! Déjà la population semble fatignée d'un excès de vigueur, et éprouver le besoin des saiggnées ansquelles on l'avait accoutumée : quatre années de conscription, c'est-à-dire plus de douze cent mille hommes, altendeut avec inpatience le jour qui leur mettra les armes à la main avec l'ordre d'inouler l'Europe, cette Europe qui recèle partout des passions prêtes à les accueillir. Une seule ville au Brésil sïnsurge, et les révoJutionnaires de toutes les nations tressaillent de joie, et espèrent que le jour de leur triomphe contre les rois est arrivé. Que sera-ce quand celle France, ce grand foyer de la révolution, quil a fallu tant d'efforts pour éteindre, ce pays qui aura été gourerné sous la direction, sous la surveillance et avec la prétendue sagesse des eabinets de l'Lurope, retournera à la fermentation et à ses principes destructeurs? 


\section{( 9 )}

par les circonstances oì elle fut décidée, par le besoin que l'Europe avait de gărantie, par l'iutrérêt même de la France, et cependant or a sersti les inconvéniens qu'on n’a pas su prévoir, au pisit que les plus iutéressés la regardent désormai- comme impossible et comme inutile pour défendre l'Enrope de la révolution de France. Quils seront douc ces moyens que l'on croyait pouvoir employer pour arrêter les explosions révolutionnaires, dont la présence des armées étrangères nous a seule garantis? Pensera-t-on que des armées plus nombreuses, placées au-dehors le loug de nos frontières, ou groupées en masses imposantes sur quelques-uns de leurs points, seront de meilleurs remparts? Pourquoi paroîtraient-elles plus rassurantes? Seront-elles plus unies sous un même ou sous un plus habile chef? Quand la ligne en sera prolongée sur une étendue trois fuis plus grande, leur action sera-t-elle plus rapide sur le centre de la France qu'elle ne pouvait l'ètre? Dira-t-on que cette occupation, changée en blocus, serait moins irritante pour l'esprit de la nation? Non: la crainte salutaire qu'elles imposaient sera moindre à proportion qu'elles seront plus éloignées, plus étendues, plus divisées, et l'irritation ru'clles pouvaient inspirer à ceux qu'elles comprimaient sera plus forte encore: d'aliord parce que les moyens de les attaquer seront plus disponibles et plus certains; ensuite parce que cet état menaçant contre cus leur paraîtra moins justifié, sa durée moins déterminée, l'union des puissances moins assurée, enfin, parce que ce systeme de compression sera réellement plus hostile. Qu'on ne s'y méprenne pas: on changerait les embarras et les inconvéniens qu'on a appris à connaitre, contre d'autres qui ne 


\section{(10)}

seraient prćférés que parce qu'on ne saurait pas les apprécier. Eufin, la pensée d'abandonner la France aux fureurs de la révolution est injuste et cruelle : elle avilirait la majesté des rois; elle effacerait l'honneur que les couronnes avaient Retrouví dans la glorieuse époque de 1814 et 1815; elle déchirerait la plus belle page de leur histoire: on ne peut pas supposer une pareille détermination.

On ne saurait donc admettre que l'Europe puisse se garantir de la révolution, si celte révolution reprend en France son pouvoir, ses forces et son activité : tous les moyens qu'on essayerait de lui opposer sont ou impossibles ou inutiles. Il ne peuty avoir d'espérance de salut que dans des efforts bien concertés, pour arrêterl'explosion au sein même de la Erance. C'est ainsi que nous sommes amenés à examiner la seconde hypothèse. Cherchera-t-on les moyens de sauver la France des fureurs révolutionnaires, pour en préserver le monde, et quels sont les moyens qu'on employera?

Si on embrasse par l'imagination toutes les combinaisons possibles sur ce sujet, on en trouvera cinq qui peuvent se présenter à différens esprits :

1. Les uns croiront peut-être éteindre la révolution en partageant la France ou l'occupant militairement.

II. D'autres imagineraient atteindre le mếme but en essayant de placer une nouvelle dynastie sur le trône.

III. Quelques uns croiront que le gouvernement représentatif a étéle grand obstacle à l'établissement du Roi, et qu'il fant le détruire.

IV. D'autres ont espéré qu'on pouvait ramener le Proi et ses ministres actuels aux principes qui peuvent consolider la monarchie. 


\section{(11)}

V. Enfin, il en est qui pensent que la revolution ne sera finie que le jour oi l'on pourra changer le système du gouvernement par le changement des ministres qui le dirigent.

\section{Partager la France ou lbccuper militai- reinent !...}

J'avoue que mon sang, tout français, se révolte à celle pensée, et que je ne pourrais la discuter politiquement, d'ailleurs les résultats de si exécrables déterminations sont démontrées dans la note du 15 août 1817 , à laquelle je me réfère $(e)$.

(e) Extrait de la note du 15 aonit 1817. Rien n'est exagéré dans les craintes que nous exprimons : l'avenir les justifiera toutes; et si les bornes de cet écrit permettaient d'en accunuler les preuves, on les porterait à l'évidence; mais quelles que soient les lecons de l'expérience, elles seront encore perdues pour les souverains de l'Europe; ils s'endormiront dans une trompeuse sécurité ; ils chercheront à se garantir de nos avertissemens plutôt qu'à șe garantir du danger, ou bien ils se flatteront d'arriver toujours à temps pour le prévenir; et ils penseront que les 120 mille hommes de l'armée d'occupation suffiront pour étouffer les mouvemens dangereux, pour comprimer l'insurrection quand elle aurait éclaté. Ils se trompent : la France sera cmbrasée avant que ces faibles moyens puissent apporter un secours suffisant pour éteindre l'incendie. La France a deux fois souffert linvasion, parce que les alliés portaient avec eux, et pour ainsi dire sur leurs drapeaux, de grandes espérances, celles d'un gouvernement qui avait pour lui de grandis souvenirs de bonheur, et des garanties d'un repos durable. Ces espérances ont été décues; et cette fois on ne les verrait plus arrirer qu'avec l'borreur qu'inspire l'ennemi qui n'a plus rieu à nous offrir en comperisation des maux die la gucre. Le prince qui les rappellerait, faute d'avoir su gouverner lui-mème, deviendrait odicus à la nation entiere; te le parti qui cherche- 


\section{Placer une nouvelle dýnastie sur le trône.}

Que deviendraient les principes de la légitinité proclanés si solennellement, que dis-je ? les principes éternels de la conservation des peuples et des trônes? que pourrait-on espérer de cette nouvelle subversion? La révolution, dira-t-on, s'accomnderait mieux d'un roi révolutionnaire. D'alsord, la révolution ne s'accommode d'aucun roi ; ensuite, la révolution peut reuverser; nats elle ne peut rien

rait sou appui dans leurs ar:ues, serait anssi ennemi que les étrangers, et serait repoussé aycc eus. Dailleurs, que seraient 20 mille hommes qui derraient occuper la France, contre le sentiment profond d'horreur qui s'stablirait contre eux dans toutes les classes de la nation? Croirait-on qu'on aurait le temps, les moyens, de rassembler encore une fois un million d'hommes pour les jeter sur cette malhenreuse France? On ne le pourrait pas dans un an; et dans vingt jours la France entiere serait un camp, une citarlelle impénétrable, dont la population cntière formerait la garnison, Se tromperait-on au point de croire qu'on pourrait eusuite, par une longue guerre, la dénembrer et partager ses provinces, et regarderait-on ce moyen comme le dernier coup à porter à la révolution? On serait dans une bien grande erreur : la France est trop compacte pour se prêter à un norcellement; des liens trop anciens et trop forts en timnent les peuples attachés. Outre cela, la première ville que l'on vondrait conquérir, le premier canton gu'on voudrait livrer comme la proie d'un des copartageans, serait bientòt pour eus une occasion de dis"corde. Enfia, quand des armées innowisrables occuperaient le sol, et guelle armée ne faurlvait-il pas pour occuper la France! quand rien ne pourrait plus déguiser a ses yeux l'horreur te son sort, alors même, dis-je, une dernière ressource, une ressource infaillible, lui resterait, la corruption de ses vainqueurs; et lá Erance révolutionnaire décomposfrait les armées victorieuses par le poison des idées rérolutionnaires. 
construire, rien établir, rien conserver ; et, si elle le $p$ urait, ne pous montrerait-rlle pas ses cuvres? Qu'elle ne dise pas meme qu'elle a fait le règne de bonaparte: il n'est pas plus sou ouvrige que les regues de Tamerlan et de Gengiskan. Ei la tnasse de la France royaliste qui avait attaché tout son espoir au retour des hommes et des principes légitimes, s'accomoderait-clle de perdre des espérances qui lui sont si chères, encore gu'elles aicut été si déçues? Quels soutiens aurait ce nouveau sonverain qui aient manqué au Roi? combien le Roi pourrait-il en trouver qui manqueraient à celui-la? C'est bien alors qu'il faudrait garnisonner la France de quelques cent mille étrangers, et les résultats d'une pareille mesure sont présentés dans tout leur jour dans la note précitée $(f)$.

On jugera lien que c'est uniquement pour completter le tableau de toutes les suppositions qui prutvent se présenter, que nous avons cru devoir discuter les questions d'un changement de dynastie, de l'occupation militaire de la France, ei du partage de ses provinces. Ce n'est pas en faisant des révolutions que l'on peut espérer de finir la révonJution, de même que ce n'est pas au milieu d’un tremblement de terre qu'on peut reconstruire. Mais, nous arrivons aux points gui mériteut une discussion plus sérieuse et plus approfondie: les nouvelles institutions politiques de la France peuvent-elles lui convenir, ctle gouveruement représentatif a-t-il été le véritable obstacle à l'ciablissenent de la monarchie en France? Suflirait-il de le détruire pour écraser cette révolution qui relève une

(f) Voyez la note précédente. 


\section{(11)}

tête si audacieuse et si langercuse? Nous allons examiner cette importante question.

\section{Détruire le gouvernement représentatif:}

Il y a des inconséquences portées à un tel degré, qu'elles ne sauraient jamais se pardonner, et quand on pose comme base d'un systeme de gouvernment deux principes qui se combattent, les résultats sont nécessairement discordans. C'est ce qui est arrivé dans les affaires de France. Les puissances alliées ont donné de la même main ct en même temps à la France, le gouvernement représentatif qui pouvait lui convenir $(g)$, et au Roile prétendu système d'équilibre entre les partis, qui devait les dominer et les détruire $(h)$. Toute l'iucertitude, toute la faiblesse du gouvernement du Roi tient à l'impossibilité d'accorder ces deux principes contradictoires. En effet, l'essence du gouvernement représentatif sous quelque forme qu'on l'établisse, est de donner légalement un organe à l'opinion publique, et à cet organe une portion de la puissance souveraine; ct il ne faut pas penser qu'on soit libre de lui donner ou de lui refuser ce pouvoir. Si l'état social est tel que cette opinion excrce une grande intluence sur les dispositions et les actes du gonvermement, on ne saurait éviter de reconnaître ce qui lui est acquis; et les lois constitutiounelles doivent régler l'exercice de ce droit, pour qu'il devienne

(o) Proclamation de l'empereur de Russie... Avril 1814.

(h) Lettre des quatre ministres au duc de Richelieu, du 20 nuvembre 1815 . Note officielle des quatre ministres, en date du 10 férrier 2817 . 


\section{( 15$)$}

l'appui du gouvernement qui l'adopte, au lieu d'étre l'effroi et l'ennemi de celui qui serait condamné à repousser continuellement ses attaques.

Mais l'expérience prouve que cette opinion ne s'exprime jamais par une seule voix, et que toutes les couleurs, toutes les nuances des opinions individuelles qui la composent et la diviseraient à l'infini, se classent naturellement, par des intérêts ou des opinions analogues, en deux couleurs qui prennent le nom et l'attitude des partis dans l'expression journalière de leurs veux, de leurs désirs et de leurs volontés. C'est ainsi que l'Angletcre a vu, depuis 160 ans, jusqu'à ce jour, la représentation nationale divisée entre les partisans de la prérogative royale et ceux des priviléges du peuple; qu'en Amérique, elle se divise en fédéralistes et antifédéralistes, et les gouvernemens de ces pays n'ont jamais imaginé de se placer au milieu de ces différens partis. Ils savaient bien que de cette manière ils ne pourraient gouverner aucun de ces intérêts, ne pourraient en contenter aucun, et par conséquent n'en trouveraient aucun qui voulut les appuyer et les soutenir; et telle est la position du gouvernement en France, placé entre les deux grandes divisions de ceux qui veulent l'établissement de la maison de Bourbon et de ceus qui veulent les conséquences de la révolution.

On voit donc qu'il existe une contradiction de premier ordre entre l'établissement du gouvernement représentatif qui constitue les partis, et la marche intermédiaire du gouvernement qui prétend les détruire. En effet, d'un côté on a appelé les diverses opinions ciui forment et lient les partis, à se prononcer avec toute l'indépendance qui caractérise la souveraineté, et d'un sutre côté on a 


\section{( 16 )}

usé tousles ressorts de l'autorité royale pour étouffer leur expression. D'une part on leur a ordonné de putler, et le l'autre on leur a commandé de se taire; d'un côté on a rassemblé les partis, on les a mis en présence, on leur a donné un champ de batitlle, dos armes, des chefs et des drapeaux; et de l'ature le gouvernement, tout seul et isolé qu'ii 'tsit, a cu la prétentiun de les désarmer et de l... disperser. Ois s'rst placé d'une part dans l'obligation rigoureuse d'obienir leur assntiment, et ie l'autre on a voulu marsher d'une naniere indépendante des intérèis des uns et des autres. Enfin, o1 a établi une forme de gouvernement qui ne peut manruer de créer des partis dans le pays mếme où il n'en existerait pas les premières traces, et on a établi en principe qu'il fallait détruire les partis dans un pays où ils existaient aussi fortement prononcés depuis trente ans. Que pouvait-il résulter de ce tissu d'inconséquences, et d'inconséquences qui s'attachaient aux bases mêmes et à l'essence dn gouvernement? Ce qui est en effet résulté, que le gouvernement, témoin inutile de la lutte qu'il ne sauroit empêcher, se trouve froissé et brisé au nilieu des chocs des deux partis ennemis, et qu'il u'a échappé à son entière destruction qqu'en appelant les secours les plus précaires et les plus dangrercus pour sá stabilité. Il n'en eût pas été ainsi, si les ninistres eussent compris la nature du gouvernement qu'ils avaient à diriger (i).

(i) Extrait de la note du mois d'août 1817. Mais, dirat-on, est-il un homme raisonnable qui put conseiller au roi et à son gotwernement de se placer dans un parti, d'en épouser les violences et les passions, d'employer la force pour faire triompher tous les intéréts des uns et pour écraser tous ccux des autres? A Dieu ne plaise que nous 


\section{(17)}

Il est certain que si la France n'avait pas tout-ifait perdu la trace de ses ancienues institutions, si le peiple avait pu supporter le joug le plus indépendant, je dirais volontiers plus absolu; si les

ne fussions pas mieux compris! mais nous disons qu'un gourernement qui est par sou essence celui des partis, qui établit une tribune où se discuteut publiquement les intérêts qui divisent la nation, ne saurait se placer dans cet intermédiaire de la faiblesse qui ne satisfait et ne rassure aucun de ces intérèts. Nous disons, et l'expérience le dit mieux que nous, que ce sage milieu qu'il faut atteindre, ce nilieu qui est la pensée de l'homme éclairé et l'intérêt de celui qui ne l'est pas, est un résultat auquel il faut arriver, mais n'est pas un moyen pour y parvenir; que lorsqu'on veut s'y placer d'ahord, on y est senI, sans force, sans appui, méprisé de tout les partis qqui luttent sur votre tète, quand vous croyez les avoir nis à vos pieds, et que le seul moyen de réussir et de confondre des intérêts divergens est de se placer au milieu de ceux qui sont les plus analogues au système qu'on préteud établir, et de les naîtriser par cette puissance qu'on exerce sur ceux qu'on commande, et jamais sur ceux qu'on combat; que c'est dans cette position et en donnant à un parti le sentiment de la conviction que l'ou est avec lui en communauté d'intérèts, qu'on peut se servir de sa force et mème de ses passions pour obtenir de lui tous les sacrifices nécessaires et la sage conciliation de tous les intérèts opposés; et pour mieux expliq̨uer ma pensée, je me servirai des expressions du prince le plus voi:in du trône, qui a dit que pour gousverner la France, il fallait se place. an milien des siens et tendre la main culu autres. En effet si, dès l'année 1815, le gouvernement s'était assis sur des principes plus positifs, il serait aujourd'hui au point de ratlacher plus entièrement, plus franchement et sans danger, les intérêts quili aurait peut-être eu l'air de froisser davantage dans le premier moment. Les royalistes, rassurés par la conviction que le gouvernement du Roi ne s'écartait pas des principes qui dans leur pensée peurent seuls le consolider, auraient été les premiers à demander que le Roi appelât à lui tous ceux qui 


\section{(18)}

propriétés étaient moins également partagées, les Jumières moins également répandues; si toute la population était moins accoutuméc à s'intéresser à toutes les actions du gouvernement, à les discuter, à lesjuger, on n'aurait pas cru nécessaire de donner à la France un gouvernement représentatif; il n'y aurait pas une tribune aux harangues, ou les partis viennent, avec toute la chaleur des passions et celle des amours-propres, aiguiser leurs armes, réveiller les intéréts qui divisent la nation, les appeler des deux côtés au combat: alors, et seulement alors, on aurait pu conseiller, adopter et suivre ce système d'équilibre, et il aurait détruit des résistances inutiles et isolées; il aurait entraîné les uns et decouragé les autres.

pouvaient le servir, et auraient volontiers accepté dans leurs rangs les nouveaux convertis à la doctrine de la légitimité. Et ne leur a-t-on pas même fait un reproche d'adopter trop facilement et sans clistinction tous ceux qui se présentaient pour soutenir avec eux la cause qu'ils défendaient? D'un autre côté, ceux qui, par leurs antécédens, se trouvaient en opposition naturelle avec l'établissement du trône, perdant tonte espérance d'expliquer leur conduite, en faisant prévaloir des principes anti-monarchiques, auraient facilement reconnu ceux qu'on aurait déclarés immuables, et s'y seraient franchement rattachés lorsqu'ils y auraient trouvé des garanties de leur avenir ; et telle a été évidenment leur disposition, dans les premiers mois de la première restauration. C'est de cette manière, et senlement de cette manière, qu'on pouvait établir la monarchie et lier la nation dans un seul faisceau. Au lieu de cela les ministres out laissé tout en question, hors la puissance révolutionnaire, devant laquelle il's se sont prosternés; aucun principe monarchique n'a été reconuu et consacré, aucune institution monarchique n'a été établie, et après 27 ans de calamités, une lutte effrayante subsiste encore entre la force qui tend à régénérer et à conserver, et la force qui ne te 々d qu'à dissoudre et à détruire. 


\section{(19)}

Aussi n'avons-nous pas été étonnés lorsque ies souverains, qui gouvernent leurs peuples avec les Jabiludes des anciennes monarchies, ont pensé que le gouvernement de France trouverait sa lorce ì tenir la balance égale entre des iutérêts opposés. Cette idée était saine; elle était dangereuse dans l'application qu'ils en auraicut fuite chez leurs peuples; elle était inutile, dangereuse, impossible avec le gouvernement qu'on élablissait on France, et nous n'avons pas attendu le résultat de l'expérience pour le déclarer formellemerit $(j)$.

Mais nous avons dû être surpris lorsque cette ponsée a été partagée par le cabinct d'Angleterre, qui devạit si bien connaître toutes les courtitions d'un gouvernement que nous avions dessiné sur leur modèle, et dont ils avaient une vicille et honorable expérience. Comment ont-ils méconnu chez nous ce qui leur aurat paru tout naturel clrez eux-mêmes, et pourquoi orst-ils si souvent donné des conseils qu'ils n'auraient pas pu adopter? Diront-ils que chez nous les passions et les partis ne sonz pas les mêmes? Il serait aussi bîn de dire que les passions humaines ue se ressemblent pas en tout temps, eu tout lieu. Et conment poursait-on penser que leur développement ue fût pas le mêne, lorsque les fornes du gouvernement, au milieu desquelles flles s'rgitent, sont aussi semblahles, et que le théatre sur lequel elles s'établissent est exactement trace sur le même dessein?

IIais, dira-t-on, l'exemple de Bonaparte ne prouve-t-il pas qu'on peutfonder un pouvoir, et un pouvoir bien terrible, sur les débris de tous les partis, et marcher audacieusement sur la tête

(j) Voyez les notes de 1816 et 1817 . 


\section{(20)}

des uns et des autres? Oıi, certes! un usurpateur, un conquérant, peut essayer, peut être encore une fois, de briser avec son épée les institutions politiques qu'il aurait créées lui-même; peut - être pourrait-il encore une fois entrainer par le prestige des conquêtes une nation ardente et mobile, et distraire ses passions par l'appât de la gloire et des récompenses. Il pourra encore armer un million de soldats, à condition qu'il puisse leur livrer l'Europe à dévorer. Et encore son pouvoir seraitil tout artificiel; et tomberait-il le jour oil ses conquêtes seraient bornées. Faut-il que Bonaparte soit encore funcste a la France, par l'application fausse et dangereuse que l'on fail de l'exemple de sa tyrannie! Toute la force de Bonaparte se trouve dans l'unité de ses conceptions, pour creer en France le despotisme militaire par la conquête étrangère. Cette force ne peut plus en être une pour la France, dés l'instant qu'elle est dans l'heureuse impuissance de désoler la terre. Comment n'a-t-on pas compris que chaque gouvernenent, suivart sa nature, avait une force qui lui était propre, et que celte force n'était autre chose que l'accord, l'harmonie des principes du gouvernement avec toutes ses conséquences et son application à tous les détails? Bonaparte créa tout pour la conquête, et son premier soin fut de briser inus les organes de l'opinion publique (k); le gouvernement représentatif doit trouver toute sa force dans le développement de l'opinion publique, dans les institutions qui penvent le favoriser, et, je dirai plus, daus l'organisation régulière des partis.

(k) Sénat à vie et soudoyé. - Corps législatif muet et payé. - Iribunat déiruit. 


\section{(21)}

Mais dialleurs quelle violence ne faudrait-il pas pour arracher aujourd'hui à la France les concerssions qu'elle a reçues du Roi? Elles ont éte consacrées par les puissances qui le replaçaient sur le trône, par l'usage qu'on en a fait, par les garanties çu'on y a trouvées; enfin, par leur alloption franche et entière de la part de ceux mêmes qui y étaient le moins préparés. Cette question ne parui pas douteuse en 1814 ; elle serait encore à discuter en 1818 ! ! . . Alors cependant on pouvait envisager les choses sous un autre point de vue. Alors il y avait une classe nombreuse d'honmes honorables qui avaient conservé les souvenirs du passé ; ils étaient embellis pour eux de toute la poésie de l'histoire, et de tout le charme que lenr prêtait le temps de leur jeunesse; avec eux on pouvait essayer, mais peut-être aurait-on essayé en vain, de replacer le trône sur les débris des bases antiques. Mais aujourd'hui ces nombreux partisans de la royauté, poursuivis et persécutés par le ministère, n'ont trouvé d’asile que dans les formes préservatrices des institutions nouvelles; ils les out franchement adoptées; ils les ont proclamées; ils les ont jurées, et ceux-li n'ont jamais juré en vain. Comment espérerait-on établir sans eux, établir contre eux, ce qu'on aurait peutêtre inutilement tenté il y a quatre ans avec leur concours?

Tout serait difficile, tout serait impossible dans une parrille tentative; on ne pourrait pas rétablir ce qu'on appelle l'ancien régime; tous les élémens en sont brisés, et la poussière même en est dispersée. On ne retronverait pas même le fantôme de ces grands corps de l'état, qui, à-la-fois défenseurs des droits de la cuuronne el des priviléges 


\section{(22)}

des peupies, se halançaient noblement dans ie cercle qui lour était tracé, et grantissaient à-lafois les libertés de la nation et l'inviolabilité du irône. Ce serait donnc un despotisme nu et hideux qu'il faudrait mettre à la place de ces belles e irréparables institutions des temps anciens; un despotisne sans force, sans institutious, sans garanties, un despotisme tel que la France ne l'a jamais counu, et ne saurait janais le supporter; un desporisme enfin qu'il faudrait maintenir par la force des armes, et qui attacherait à la légitimité tous les inconvéniens et tous les malheurs de l'usurpation. Un pareil gouveruenent répugnerait à la France entière, et répugnerait bien plus encore au noble caractère des princes légitiuses.

Qui oserait dans ce système, se charger de demander à la France les sacrifices que de dures circonstances lui ont imposés, quand elle peut à prine en trouver les moyens dans le concours de sa volonté et de ses efforts? Que deviendraient les ressources du crédit aurjuel elle est condannée dcpuis long-temps? L'impossibilité d'adopter une semblable mesure paraît portée à l'évidence.

Et en faveur de qui prélendrait-on exécuter une pareille subversion? Ce ne serait pas dans les intérêts du pays, qui ne trouverait plus dans le gouvernement légitime ancun gage de stabilité; ce ne serait pas dans les intérêts de l'Europe, qui s'engagerait à soutenir par la force le gouvernement gu'elle aurail imposé par la force; ce ne spraic donc que dans l'intés êt de quelques noms propres, qui croiraient ausi se maiutenir plus facilement an pouvoir. Aussi ignorans dans leurs idées de le conserver, qu'ils le sont dans les moyens de l'exerce:, les ministres seraiert bien étonLés eur-mêmes de 


\section{(23)}

découvrir que ces formes constitutionnelles, qu'ils regardent comme des entraves, étaient leurs seuls appuis, et de se trouver plus faibles à mesure qu'ils auraient brisé ces utiles barrières.

En effet, si, depuis trois ans qu'ils l'exercent, les ministres avaient compris un seul instant le gouvernement représentatif, n'en auraient - ils pas éprouvé les avantages au lieu de n'en ressentir que les inconvéniens? Atlaqués par des partis, n'auraient-ils pas trouvé des partis intéressés à les défendre? Si la tribune retentissait contre eux de paroles ennemies, n'auraient-ils pas eu la tribune pour défendre leurs aclions, leurs motifa, leurs intentions? Que de ressources n'auraient-ils pas trouvées, s'ils avaient su se lier aux défenseurs des principes de la monarchie, ressources qui sont toutes refusées aux ministres du pouvoir absolu.

11 restera donc démontré, à tout esprit judicieux, que toutes les tentatives que l'on ferait pour détruire en France le gouvernement qu'on y a établi, seraient dangereuses; que ces formes constitutionnelles sont les mieux adaptées aux circonstances où la France se trouve placée; qu'elles conviennent à l'esprit des hommes et des temps; qu'elles sont un pacte raisonnable entre les institutions anciennes, qu'on ne saurait rétablir, et les théories de la révolution, qu'il est si essentiel de détruire; qu'au lieu de ranimer l'esprit de révolution, elles auraient pu contribuer plus que toutes les autres à le décomposer; enfin, que bien loin d'être l'écueil contre lequel se brise le gouvernement du Roi, elles auraient au contraire puis. samment servi à son rétablissement, si on ne les avait pas compliguées par un système absolument contradictoire, et si les ministres, qui ont été 


\section{( 24 )}

chargés de les conduire, en avaicnt su coniprendre la nature et les condutions. La première de ces conditions eút été de ratlacher à eux tous cenx qui veulent avec passion établir la monarchie légitime, et d'embrisser les principes qui peuvent la consolider. Nous allons exposer ce qui a été fait dans ce graud intérêt.

\section{Ramener le Roi et ses Ministres actuels aux} principes qui peuvent établir la monarchie.

Nous avons hien expliqué, dans les notes de 1816 et 1817 , par quel concours de circonstances le Roi et les miuistres actucls avaient été entraînés hors de toutes les doctrines monarchiques, et dans des directions tout-à-fait opposées à l'établissement du trône. Tous ceux qui ont jugé cet entraînement et la possition où il metrait la France, se sont réunis dans l'espérance que des intérêts micux entendus rapprocheraient le Roi et ses ministres des partisans de la royauté. Ils ne doutaient pas que le gouvernement ne fùt effrayé de voir que tous les sacrifices qu'il faisait à la révolution ne pouvaient pas servir à la rattacher au trône légitime, et qu'elle se servait au contraire de ces concessions pour élcver contre lui un front plus ennemi; ils ont enfin espéré que le Roi et ses ninistres feraient cesser cette guerre injuste et impolitique qu'ils conduisaient contre les bommes monarchiques, et les rattacheraient à leur défense. Cette marche était aussi facile qu'clle était d'un succès assuré : les royalistes, malgré le sentiment de leur force dans la population et dins la propriété, ne mettaient aucunes conditions personnelles pour cimenter cette 


\section{( 25 )}

union et conclure cette paix désirée. "Ratlachez) vous, disaient ils aus ministres, aux seuls prinx) cipes qui puissent établir la monarchie; renoncez ) à vos haines contre ceux qui veulent franchement ) la défendre. A quoi nous servira votre pouvoir, ग) à quoi nous servira votre filélité, si, emportés ) par d'aveugles passions, vous ne savez pas faire ) usage de l'une, et que vous repoussiez l'autre? ग) Qu'avez-vous à espérer des amis que vous cher) chez dans les partis de la révolution! ils avouent ) hautement le projet de renverser le trône; es) pérez-vous quelque sûreté pour vous sur ses dé) bris? Qu'avez-vous à craindre de nous? tous nos ) sentimens sont pour le Roi ; les ministres de son J) choix seront nos chefs naturels, à l'instant où ils ) voudront embrasser franchement avec nous les ) principes et les doctrines qui peuvent assurer à ) notre malheurcuse patrir, et à nos cufans, le bien) fait de la monarchie légitine solidement établie.)》 Les ministres, sourds à cette voix de paix et de conciliation, répondaient : "Nous voulons bien ) que vous veniez vous ranger sous nos bannières ) désertes; nous acceptons une force que, malgré )) tous nos efforts, nous n'avons pu briser; mais ) nous l'acceptors pour la détruire, pour qu'elle ) nous serve aveuglément, que vous abandonuiez ) vos principes, vos sentimens, votre conscience, ») et vous laissiez guider au gré de nos caprices. ) Toutes les puissances de la terre réunies n'auraient pu obtenir un pareil sacrifice, et faire que les royalistes devinssent tout-à-coup les soldats de la révolution; et cependant, voila ce que demandait un ministère sans force, sans pouvoir, saus conception.

On a bien pu juger alors par quelle fatalité nous 


\section{$\left(26^{\circ}\right)$}

nous serions entraînés : les ministres qui avaient été si empressés et si souples pour obtenir l'assentiment des étrangers, dans toutes les circonstances qui servaicnt leurs passions, étaient sourds cette fois à la voix de tous les cabinets qui leur répétaient: "Vous ne pouvez soutenir le Roi qu'en vous rat-

) tachant à tout prix la masse de la nation, qui veut ) le conserver, et en renonçant ì l'appui dange) reux de ceux qui veulent le renverser?"

Quelle espérance peut-il rester que des hommes aveuglés à tel point que les invitations des alliés, les conseils de leurs amis, les supplications des gens de bien, le sentiment des maux de la patrie, la vue des dangers quila menacent, r'ont pules ramener, puissent jamais revenir à de meilleurs sentimens?

Mais par quelle raison attache-t-on une si grande importance à maintenir à la tête des affaires quelques hommes qui r'y ont été placés que par l'embarras du choix? Mais, sur onze personnes qui ont passé au ministère depuis cette époque, il n'en reste que trois de celles qui y ont été d'abord appelées. Croirait-on qu'ils doivent être plus fidèles que d'autres à suivre la direction qui leur fut tracée à cette époque? Mais cette direction a tellement varié qu'ils ont d'áhord été royalistes; ensuite, ils ont passé à une prétendue modération; à présent ils sont dans la révolution, et ils y seraient encore davantage si la révolution voulait les adopter aussi franchement qu'eux-mêmes en adoptent les principes. Ainsi ce serait un élrange abus de croire qu'en soutenant le ministère, on soutient lès mênes hommes et les mêmes principes : ce serait un genre de conséquence bien singulier que celui qui conduirait ainsi à croire que l'on persiste lorsqu'on marche dans des contraires. 


\section{(27)}

Mais, sil'on cst convaincu, comme on doit l'être, que la réunion de plusieurs millions de royalistes est nécessaire an gouvernement du Roi, et peut scule le consolider, comment pourrait-on imaginep que ce lien politique puisse se former autrenent que par une confiance mutuelle, confiance si difficile à établir après une lutte si longue et si acharnée? Il est donc mulheureusement vrai de dire que les ministres actuels sont les moins propres à former celte réunion; qu'ils y sont peu disposés, parce qu'ils sont entrâiues par leurs passions, et que ce moyen de ramener les affaires à une meilleure direction est un des plus difficiles et des plus incertains.

On voit, d'après cela, combien d'efforts ont été tentés pour rattacher les intérêts des ninistres à ceus de la monarchie, combien d'espérances ont été trompées, combien il en reste peu d'obtenir ce résultut récessaire au salut de l'Europe, combieu il serait dangereux de placer auprès de si grands intérèts les misérables intérêts de quelques hommes. $E_{t}$, en effet, importe-t.il plus de conserver tel nom dans le ministere de France que d'assurer de si importantes destinérs? Ceci nous conduit naturellement à examiner s'il n'y a pas de moyens plus simples et plus certains d'arriver à celle réunion désirée de tous les intérêts monarchiques et européens, et à traiter la dernière question.

V. Changer le système du gouvernement par le changement an ministère qui le dirige.

Cr n'est pas seulement dans le gouvernenent représentatif qu'on a éprouvé que le changement des 


\section{( 28 )}

hommes qui conduisaient les affaires d'un pays, était le moyen le plus simple, le plus naturel et le plus certain de changer le système d'administration qu'on reconnât faux ou erroué. En effet, quoique la direction des affaires publiques repose bien plus sur les principes que l'on adopte que sur les hommes qui les conduisent, cependant il est ordinaire de voir les hommes s'ilentifier à des principes par des antecrédens, par leur conviction ou par leurs intérêts, de telle sorte qu'ils n'ont plus la possibilité de choisir d'itutres voies, de professer et d'appliquer d'autres doctrines. N'a-ton pas vu, sous tous les gouvernemens, changer les preniers agens du pouvoir, lorsqu'on a voulu changer le systeme politique extéricur, ou les principes de l'administration intérieure?

Si cette règle est admise dans les états où le souverain réunit en sa personne la puissance suprême, où les ministres ne sont et ne peuvent être que les instrumens de ses volontés, combien, à plus forte raison, la trouveraton élablie dans le gouvernement constitutionnel, où les ministres sont au contraire les conseils avoués du souverain, soumis à une responsabilité qui les autorise à suivre d'un commnn accord une marche que le prince doit approuver, mais dont il ne dirige pas lui-même toutes les impulsions? Dans un gouvernement qui doit lier à lui une masse d'intérêts qui se présentent sous l'apparence et la forme des partis, et qu'il est absolument réccssaire d'unir à l'administration par la confance que leur inspirent les hommes que l'on appelle à la composer, cette confiance ne saurait s'établir au moment même où ils arrivent au pouvoir; il faut qu'elle ait des bases plus anciennes et plus profondes, qu'elle snit fondée sur le sentiment qu'ils ne sauraicut abandonner les principes et les intérêts généraux qui unissent cette masse. 


\section{( 29 )}

En effet, que peuvent dans le gouvernement constitutionnel des ministres qui ne porlent pas avec eux et n'attachent pas à la défense de la conronne la puissance de l'opinion publique, exprimée par une de ses voix prépondérantes, ou, pour parler plus simplement, la puissance d'un parti? Incertains dans leur marche, ils ne peuvent embrasser ni un système de gouveruement, ni un cosemble d'opérations; jouets livrés aus vents de tous les partis, ils restent sans pouvoir, parce qu'ils sont sans appui; et un gouvernement sans pouvoir est un édifice sans base qui s'écroule de lui même eı couvre de ses débris la terre qui le portait.

Et qu'on ne nous reproche pas de nous jeter ici dans des théories abstraites : il est bien temps d'apprendre les conditions du gouvernement constitutionnel que l'on veut établir; il est bien temps que ceux qui veulent exercer de l'influence, apprennent comment on doit la diriger : l'ignorance de ces conditions et la ré-istance qu'on a mise à les adopter, sont les seules causes de cette incertitude, de cette vacillation des affdires en France, et de la nullité de son gouvernement.

Ainsi, sil est impossible de ne pas admettre que le changenent des personnes qui composent le niuistère soit le moyen le plus certain de changer le systeme faux et dangereux du gouvernement, il est aussi impossible de nier que ce changement serait inutile dans le gouvernement représentatif, s'il n'amenait pas à la tôte des affaires des hommes forts de la contiance d'un des deux partis qui expriment l'opinion publigue. Tous ceux quion voudrait appeler dans cette position et qui u'auraient pas des liens politiques antérieurs avec l'un ou l'autre, ne seraient pas propres à les former, parce que l'exer- 


\section{( 30$)$}

cice du pouvoir, qui nécessairement dans sa marclie est obligé de fouler des intérêts, éveille bien plus la méfance et l'opposition, qu'il ne pcut servir à serrer des uœuds de confiance et d'harmonie.

Tels seraient les obstacles insurmontables que trouverait un ministère nouvean qui, par les noms propres qui le composeraient, ne s'rttacherait pas, dès sa formation, une des deux nuances d'iutérêts généraux qui divisent l'opinion. Il ne faudrait pas trois mois, ou la présence d'une Chambre, pour juger l'inutilité d'uur pareille modıfication, et pour qu'un pareil ministère fût dans une position aussi faible, aussi nulle, aussi humiliante que le ministère actuel.

Mais, dira-i-on, si un nouveau ministère, composé d'hommes qui n'ont pas une couleur prononcée, et dont les noms, s'ils n'ont aucun soutien, n'eveillent du moins aucune ininitié, s'engageait à embrasser les doctrines soutenues par un des partis, ne parviendrait-on pas à former une admiristration impartiale, et qui serait cependant soutenue de toute la force d'un parti? Etrange abus d'un faux esprit qui croirait arriver mieus au but par des chemins incertains, difficiles et tortueus, que par la route simple qu'indiquent le bon sens et la nature des choses.

D'abord ce ministère, qui arriverait sans que des liens préalables l'urissent à un parti, aurait à traverser un temps d'épreuves plus ou moins lorıg, avant d'avoir obtenu cette confiance qui doit faire sa force; et la confiance est si délicate gu'il pourrait échouer dans cette tentative. S'il y réussissait, c'est parce qu'il aurait cessé d'être inspartial; en s'attachant les intérêts des uns, il aurait sûrement aliéné ceus des autres: surveillé ayec inquiétude 


\section{(51)}

par le parti auquel il se serait nouvellement aliié, il n'obtiendrait jamais de lui le sacrilice de ses intérêts particuliers, souvent nécessaire à une sage conciliation des intérêts généraux; sacrifices qui seraient facilement accordés à ceux qui seraient liés à ce parti par des noeuds plus forts et plus anciens. Outre cela, ces hommes qui auraient adopté des doctrines qui ne seraient pas exactement les leurs, auraient toutes les difficultés à les appliquer dans tous les détails; les principes qui lient un parti sont dans son intérêt, dans ses habitudes, dans ses affections, dans tous ses sentimens, et il faut tout cela pour se trouver d'accord dans tous les temps et dans toutes les circonstances. Ceus qui n'auraient pas traversé les mêmes antécédens, épouséles mêmes intérêts, ne pourraient, avec la meilleure volonté, avec la plus grande franchise, posséder cette conscience d'un parti, qui seule peut éclairer dans l'application journalière de ses principes: ils n'en sauraient pas les doctrines et les moyens, et seraient sans cesse obligés de consulter ceux qui les connaissent, et qui, par leur assentiment, entraîneraient celui de la masse qui est accoutumée à les suivre: c'est-à-dire, qu'on aurait deux ministères, l'un sans appui, dès qu'il se séparerait un instant de l'autre qui aurait la véritable force; l'un obligé de consulter l'autre qui imprimerait la véritable direction, l'un en apparence, l'autre en réalité ; et qui pourrait soutenir long-temps une pareille division?

Nous croyons avoir porté jusqu'à l'évidence ces vérités essentielles, que, pour changer un système d'administration, il faut changer ceux qui le dirigent; que, dans le gouvernement constitutionnel, le pouvoir est nul s'il ne s'appuie sur un 


\section{(52)}

des organes de l'opinion publique qu'on appelle partis; enfin, qu'on ne peut atiacher au gonvernement un parti d'une manière utile, certaine et irrévocable, qu'en plaçant dans l'administration ses chefs naturels, les hommes de son entière confialce. Mais il reste encore à discuter une importante question: "Comment dans un changement de ) Ststeme le gouvernement se décidera-til pour 》) choisir le parti qu'il doit appeler à son soutien?") Ici toutes les opinions se ressentent des mtérêts : les uns disent que c'est le plus fort en nombre dans la nation, el tous les deux peut-être auront la prétention de décider en leur faveur une question qui reste éternellenent indécise, jusqu'à ce qu'elle soit décidée par des élections libres et à l'abri de toute influence. Les autres soutiendront qu'au lieu de la force du nombre, le gouvernement doit s'appuyer sur la force de la propriété; enfin, chacun fera valoir ses avantages pour faire prédominer ses principes. Mais en France, comme dans tous les autres pays oì l'on a usé du gouvernement constitutionnel, la question est plus simple, et le gouvernement n'a jamais été appelé d'une manière indépendante à faire un pareil choix, et partout il s'est trouvé, par la nature des choses, un parti plus intéressé à sa défense et à son maintien, et un autre plus disposé à l'atlaquer, soit pour le renverser, soit pour en obienir de plus grandes concessions: enfin, il a nécessairement pour ennemis les intérêts qu'il a froissés par son établissement, et pour anjis les intérêts que son étallissement a relevés : il n'a donc pas de choix il faut qu'il s'appuie sur ceux qui veulent li. soutenir, pour se défendre de ceus qui veulent le renverser.

Mais on u'arrive pas à la solution đae cette gnestion, 


\section{( 55$)$}

sans éprouver le besoin de se prémunir contre l'huée qu'il s'y rattache des questions personnelles, que toutes les affaires de France, que toute l'opposition des grands intérêts de ce pays, que les principes qui divisent les partis, se réduisent à quelques misérables prétentions déçues, et à quelques ambitions trompées.

Nous l'avons déjà dit, les royalistes qui ont été appelés dans diverses occasions à traiter de la réunion de leur parti au ministère, n'ont jamais admis la possibilité qu'il y eût pour eux un prix à ce traté. Ils n'ont demandé ni places ni honneurs; ils ont repoussé de pareilles conditions quand elles leur ont été offertes. Ils savent mieux que personne qu'il n'y a point de place à désirer dans une maison qui brûle, et que la plus dangereuse dans un vaisseau brisé par la tempête, est celle de capirtaine. Les principes de leur opposition sont dans la connaissance du mal qu'a fait à la France le système qu'on a saivi : et quels sontceux qui ont été mieus placés qu'eux pour juger l'excès de ce mal? Ils se dévoucraient peut-être à le réparer par ce sentiment du lien et de l'anour de leur pays, qui les a soutenu dans la terrible situation où ils ont été placés; mais ils n'ront jamais au-devant d'un fardeau dont ils conuoisseut mieux que d'autres la pesanteur: les plus éclairés sont ceux dont on ohtiendrait le plus difficilement le concours, au momerst où on voudrait le leur demander.

Celte vérité une fois ardmise, ề elle ne peut être revoquée en doute que par la mauvaise foi la plus insigne, nous pouvons exprimer notre étonnement qu'on n'ait jamais admis, comne la marche la plus simple et la plus naturelle, au milieu des difficultés que présentele gouverneneat intérieur de la France 


\section{( 34$)$}

et des dangers qui menacent son établissement, d'en confier les destinées à ceux qui, par leur antécédent, par tous les sentimens de leur ame et de leur conviction, ne pouvaient avoir d'autre intérèt, d'autre avenir, d'autre abri que celui du trûne reconstruit. Cependant les combinaisons les plus extraordiuares sont celles qui ont paru préférables; on a voulu appuyer le trône sur tous ceux qui avaient été opposés à son rétablissemen: : tous, jusqu'aux régicides, ont passé au pouvoir, et ce calcul a parule plus habile, parce qu'il étaic le moins avoué par la raison et le bon sens. Enfin, toute celte sagesse des honmes a conduit à prendre les ouvriers les moins propres à l'ouvrage qu'on voulait leur confier. Imagineraiton jamais de remettre à unzèlé protestant la défense des dogmes catholiques; et croirait-on la religion en sécurité sil'on choisissait des athées pour la soutenir? Il en est de même des opinions politiques: il n'est pas plus donné à un royalistc d'apprendre et d'employer les moyens révolutionnaires ou même de faire cle la république, qu'il n'est donné aux honmes de la révolution el du despotisme de faire de la monarchie constitutionnelle.

Quelque simple que soil cet aperçu, nous ne devons pas dissimuler les objections qu'on lui oppose, objections le plus souvent intéressées. Nous les avons toutes discutées dans la note du mois d'août 1817 , nous y renvoynns nos lecteurs pour éviter une fastidicuse répétition $(l)$.

(l) Fxtrair de la note du mois d'aoñt 18,y. - Les royalistes nélaient pas assez nombreux dans la nation, assez for.s pour soutenir le tróne. Mais si les royalistes sont seuls à vouluir le soutenir, est-ce en les divisant, en les atta- 


\section{( 35$)$}

Il est cependant vrai que les royalistes, placés comme ils, le somt sur le terrain de la constituton, sont les seuls qui puissent sauver leur pays, les seuls qui puissent soutenir le trône et conserver

quant, en les dépouillant de toute autorité, qu'on parviendra à le maintenir? Il serait plus vrai de dire que les royalistes n'étaient pas assez décidés à soutenir le ministère.

Les royalistes sont trop faibles pour soutenir le gouservement du Pioi, qui s'appuyerait str eux; mais, d'un autre côté, ils disent que les royalistes, dépouillés de toute la prépondérance que leur donnerait le gouvernement, sont assez forts pour l'entraver et l'arrêter à chaque pas. Les royalistes sont trop faibles, et tous les efforts des ministres s'emploient ou plutôt se perdent à les diriser. En effet, toutes les parties de l'administration, les intérèts politiques, tout est négligé dans le gouvernement, et, depuis dis-lıuit mois, il n'y a eu d'action que pour la guerre de passion qu'ils font aus royalistes. Cette résistance d'un parti contre lequel on a tourné son chef naturel, le seul qu'il puisse reconnaître, prouve quelle aurait été sa force pour asscoir et consolider l'autorité royale bien autrement qu'on n'a pu le faire par la série d'inconséquences dans laquelle on est entraîné. De quoi se compose donc la force des royalistes? Elle se compose de la plus grande partie des propriétaires territorianx dans les classes les plus importantes $\left({ }^{*}\right)$, de tout le clergé de France, de tous ceux qui conservent des principes religieux, de populations entières et nombreuses dans les provinces de l'Ouest et du Midi. Outre cela, la conséquence immédiate da retour du Roi avait été de réunir à eus touse une masse incertaine et faible de la nation, comme dans toute nation du monde, qui est prête à olvéir à la direction que lui imprime le gouvernement, mais qui ne fera jamais rieu pour le sou-

(*) Sur izon propriétaires payant $1000 \mathrm{fr}$. d'impôt, les neuf dixiènes sont royalistes dans un sens opposé au ministère. Sur 45.000 payant $500 \mathrm{fr}$., plus de la moitié partage les mèmes opinions. Sur 120,000 payant $500 \mathrm{fr}$., les royalistes sont en zombre inférieur. Sans la division que le faux système du gouveruement a établie, ils seraient les plus nombrenx dans cette dernière classc. Les proportions sunt presque contraises dans les fortunes nobilières. 


\section{( 36 )}

dans leur intégritéles priviléges acquis par le peuple. Serait-ce aux révolutionnaires qu'on pourrait confier ce double dépôt? Leur haine naturelle est contre le pouvoir légitime, leurs habitudes sont la

tenir. La multitnde elle-même était bien plus disposée à comprendre et à embrasser les principes simples, positifs, certains, des royalistes qui leur présentent un chef visible et une doctrine complète, que toutes ces manies métaphysiques, toutes ces hérésies politiques qui embrouillent toutes les idées, qu'ils ne sauraient saisir, et auxquelles ils ne peuvent s'attacher. Il est facile de comprendre quel a dủ être et quel a été l'effet des prédications du ministère du Roi, qui leur a crié, pendant plus d'un an, qu'il ne fallait pas être royaliste, qu'il fallait se méfier de ceux qui l'étaient, que cétaient des ennemis qui nourrissaient des inteutions perfides. Surpris de ces inconséquences dont ils ne pouvaient pénétrer les motifs, les hommes qui composent cette masse sont demeurés incertains, sans atlachement, sans confiance pour un gouvernement qui emploie toute son action à diviser, à combattre, à détruire ses soutiens naturels. Et, en effet, que dirait-on des ministres d'Angleterre si d'alsord ils avaient mis toute leur politique à tenir une balance prétendue égale entre les rebelles deSpafields et ceux qui veulent soutenir le gourernement; s'ils eussent ménagé, protégé et encouragé ceux qui étaient le plus disposés à se mettre à la tête des mouvemens de Londres et des provinces, et s'ils en étaient enfin arrivés au point de rechercher l'appui et l'alliance des insurgés contre ceux qui veulent maintenir l'État? Voilà cependant ce qu'ont fait les ministres de France.

Mais, dira-t-on, quels sont done, parmi les royalistes de Trance, les hommes asses éprouvés dans les affaires pour qu'on puisse leur confier des destinées aussi importantes? Ces hommes se trouveront noubrens parmi ceux qui n'ont pas d'autre intérêt que l'établisseunent de l'autorité rovaie, parmi ceux qui n'ont aucun antécédent qui les gêne et qui les domine. Ces hommes, dis-ie, se trou eront nombreux, et la confiance publique les désignera assez quand on sera arrivé a u point d'annoncer sans ciétour que c'est la monarchie, et non la révolution, u'on veut consolicier; et je demande, à mon tour, à ceux qui voudraient faire peser sur les roya- 


\section{(57)}

violence et l'arbitrairc. Serait-ce anx ministres aetuels? Ils n'ont pas compris un seul instant la doctrine du gouvernement représentatif: ils ont cherché tous les jours à se soustraire à ses règles, et ont tour-à-tour appelé à leur secours les souvenirs inappréciables de l'ancien régime, les mesures odieuses du despotisme militaire, ou les dangereuses doctrines des clubs révolutionnaires.

Quelle serait aujourd'hui la situation de la France et de l'Europe, si les hommes d'honneur et de loyauté, liés par leurs antécédens, je dirais même par leurs passions, au soutien de la maison régnante, avaient dirigé depuis trois ans les affaires de leur pays? Au lieu de briser les liens de la morale et de la religion, on les aurait vu soumettre peuà-peu le pays au joug des principes morauz et religieux, et ils auraient été facilement adoptés comme les conséquences les plus immédiates du gouvernement légitime: au lieu de chercher leur appui dans les passions d'un vain peuple, et de les animer contre les puissances alliées auxquelles la France a dû l'espérance d'un meilleur avenir, ils auraient, par leurs préceptes et leur exemple, fait respecter les couronnes alliées; sentiment qu'il était facile d'inspirer, quand on aurait su faire

listes seuls le résultat infaillible d'un gouvernement qui a détruit toutes les existences, toutes les réputations; je leur demande quel est celui des ministres actuels qu'une seule voix eût désigné, il y a deux ans, comme capable d'en remplir les fonctions, et que l'opinion regardât comme préparé à y parvenir. Ce ne sont donc pas les honmes qui nous inanquent? Il n'est pas difficile d'en trouver en France un grand nombre qui porteraient dans les affaires publiques plus de raison, de force et de discerne'ment que ceus qui les dirigent aujourd'hui. 


\section{(58)}

respecter la couronne de France. Les avantages des munvelles institutions seraient appréciés, parce qu'ou les antrait strvies et comprises; ct au lieu de les ébranler par l'ignorance et la mauvaise foi, elles scraient établies asce tontes leurs conséquences et dins tonte leur force. Le trône et la monarchie seraienc pusés sur des bases inébranlables, au lieu d'être jotés sans appui et sans soutien au milieu de passions eunemies; la révolution serait repoussée dans quelques abstractions, méprisées, et dans une opposition pen dangereuse; l'Europe rassurée compterait sur nous, et ne pourrait plus nous demander des gages de notre tranquillité; elle les tronverait dans notre gouvernement.

Et qu'on ne nous accuse pas de tracer ici un tableau fantatisque: tels étaiest les fruits naturels de la restauration; il a fallu bien plus d'efforts pour les dénaturer que pour les recueillir : voilà ce qu'on devait obtenir et ce qu'on pourrait peutêtre obtenir encore.

Nous sommes arrivés à ce point dans le développement des principes du gouvernement représcritatif, et de leur application à la situation de Ja France, ou il est naturel de penser qu'on nous demusdera de quelle maniere nous concevons que les puissances alliées pourraient diriger leur inflarnce puur amener d'aussi heureux résultats. "Que peuvent, nous dira-on, les cours alliées "sur des déterminations qui doivent partir de la ) seule persuasion, de la seule volonté du Roi? ग) Peuvent-elles, même dans un intérêt qui serait

3) bien recounu, intervenir d'une manière déci-

) sive dans des questions du gouvernement inté3) ricur? Une disposition aussi contraire aux prin) cipes les mieux établis ne ferait - elle pas un 


\section{( 59$)$}

) mol plus grand que tout le bien qni pourrait en ) résulter?")

Celte objection est précisćment la même que celle qu'on faisait, en 1814 , contre le retablissement de la maison de Bourbon: on disait, à Langres, à Troyes, à Chàtillon, que les priucipes du droit public prohibaient aus puissances tonte disposition sur le gouvernement intérieur du pays, et qu'on ne saurait, sans manquer a ces pribcipes, renverser Bonaparte et rétublir la famille royale. On oubliat alors, comme on oublie aujourdhui, que la révolution qui attaque toutes les couronnes, a établi entre elles une nouvelle solidarité; que l'intérèt le leur défensc légilime, qui est en même temps le premier intérêt de leurs peuples, leur commande d'arrèter l'incendie partout où il peut éclater; qu'aucun principe établi ne peut infirmer ce graud principe de légitime défense : et ne voit - on pas dans le droit commun les principes mêmes de la propriété, sur lesquels repose la société entière, se modifier quand il s'agit de se préserver d'un incendie ou des ravages d'un torrent? Enfin, permettrait-on à un citoyen de mettre le feu à sa maison, dans l'intention de brûler toute une ville?

Telle est la situation des puissances alliées en présence de la révolution qui renaît chez nous, et c'est en ce sens qu'ont été stipulés les traités qui lient ces puissances entre elles, et qui établissent tous lecirs rapports avec la France ( $m$ ).

(m) Voyez Traité de Chaumont, du 1. ${ }^{\text {er }}$ mars 1814 ;

Convention de Paris, du 23 avril 1811 ;

Motifs du traité de Paris, du 3o mai 1814;

L'Acte du congrés de Vienne, du 19 juin 1814. 


\section{(40)}

Toutes en ont admis depuis quatre ans, qui étaicut jusqu'alors inconnus, parce qu'un nouvel ennemi el des armes offensives nouvelles demandaient de nouveaux moyens de défense. On ne

Le motif du traité du 20 novembre 18.5 n'est-il pas textuellemeut expliqué en ce sens : Le desir de consolicler le maintien inviolable de l'culorité royale et la remise en activité de la Charte constitutionnelle, et de détruire les funesles effets de la révolution et du systène de conquêtes.

Dans le même traité, l'occupation, conme des mesures de préraution et des garanties temporaires pour les états voisins?

Dans le traité du 20 novembre entre les quatre cours coa. lisées, elles disent que le repos de l'Europe est essentiellement lié à l'ordre des choses en France, fondé sur le maintien de l'autorité royale et de la Charte constitutionnelle, et qu'elles veulent employer tous leurs moyens pour que la tranquillité générale, oljet des rous de l'humanité et le but constant de leurs efforts, ne soit pas troublée de nouveau. Elles préroient même que les mêmes principes réroIutionnaires pourraient encore déchirer la France et menacer le repos des autres états; les hautes parties contractantes reconnaissent solennellement le devoir de redoubler leurs soins... .... et de concerter entre elles et avec S. M. T. C. les mesures qu'elles jugeront nécessaires pour la sûreté de leurs états respectifs et pour la tranquillité générale de l'Europe.

Elles ont même prévu, dans le mème acte, des réunions, consacrées aux grands intérêts communs et à l'examen des mesures, qui, dans chacune de ces époques, seront jugées les plus salutaires pour le repos et la prospérité des peuples, et pour le maintien de la pais de l'Europe.

Dans la note de la même date, adressée par les ministres des quatre cours alliées à M le duc de Richelieu, ne disentils pas formellement que l'oljjet de ce traité a été de donuer aux principes consacrés par ceux de Chaumont et de Vienne, l'application la plus analogue aux circonstances actuelles, et de lier les destinées de la France à l'intérêt commun de I'Europe? En effet, toute cette note porte sur les principes du gouverzement intérieur de la France, et pourrait être citée loute entière comme preuve que les puissances n'out jamais compris autrement leurs raprorts asec la France et les liens que leur ont donués ees rapports. 


\section{(41)}

peut donc plus, sans mauvaise foi, établir que la marche intérieure du gouvernement doive rester hors de toute influence, hors de toute considération de l'Europe, qui n'a rétabli ce gouvernement que pour assurer le repos public et la sécurité générale. La France rentrera dans l'entière indépendance de ses dispositions intérieures, lorsque les hommes qui dirigent les affaires ne seront plus en hostilité avec les principes de la société européenne.

On voit dans quelle erreur on tomberait si on s'arrêtait à celte objection, fondée sur le principe rigoureux et excessif de l'indépendance des états, pour se refuser à exercer une heureuse influence sur les déterminations du Roi de France, à l'effet de changer le cours des choses qui conduit évidemnent au triomphe de la révolution.

D'ailleurs il s'agit bien plus, en ce péril commun, d'ćclairer la volonté du Roi, que de lui en imposer une: l'assentiment que les cours alliées ont trop long-temps donné à la marche du ministère, a été la première et presque la seule raison de J'entraînement du Roi. On ne peut pas douter que l'intervention franche et ouverte des puissances alliées ne suffise pour l'éclairer sur ses vrais intérêts $(n)$, et le ramener à des idées plus simples et plus saines.

Dans la note officielle des quatre ministres, du 10 février 1817 , relative à la diminution de l'armée d'occupation, ils disent que le Roi cherche, de concert avec les autres puissances, les moyens les plus efficaces de consolider l'ordre intérieur en France, et d'associer sun royaume au système de bonne intelligence et de pacification générale, interrompu par les troubles qu'on venait à peine d'arrèter.

(n) Ils pourront seuls (de noureaux ambassadeurs) appro- 


\section{(42)}

Outre cela, dans toutes les négociations qui se traitent et celles qui vont s'ouvrir, soit pour terminer les liquidations étrangères, soit pour décider l'évacuation du territoire, il serait bien facile de placer en première ligne ce premier, ce grand intérêt, qui est au-dessus de tous les autres pour les conronnes et pour les peuples. Les puissances alliées n'ont-elles pas de garanties à nous demander? Ne doivent-elles pas les modifier sur la sécurité que leur donnerait notre situation intéricure? Ne sont-elles pas autorisées à proportionner leur exigence à nos dispositions? Ne peuvent elles pas nous forcer d'être heureux, et nous récompenser d'avoir su le devenir? Et si elles peuvent être aujourd'hui nieux éclairées sur la nature des intérêts qui divisent la France, sur son gouvernement, sur la position entièrement fausse du ninistère au milieu de ces intérêts et de son gouvernement, si elles ue perdent pas de vue le but de tous leurs efforts, le motif de tous leurs traités depuis quatre ans, elles ne laisseront pas échapper l'occasion de négociations importantes, sans en profiter pour amener un autre et meilleur ordre de choses.

Les souverains alliés ne peuvent-ils pas dire au Roi : "Nous sommes disposés à seconder de tous

cher du Roi des lumières que les vrais Français voudraient lui faire parvenir, et qu'il repousse comme partiales, comme incommodes. Et à quoi lui serviraient-elles, en effet, sïl n'étail pas sûr d'en pénétrer ses co-alliés? mais, au contraire, ce doute quils lui exprimerout sur les moyens employes jusqu'à ce jour pont fonder la monarchie en France, sera le premier donte quil se permettra lui-mème : leurs appréhensions seront pour lui des eraintes salutaires; car elles seraient inutiles si les souverains alliés ne les partageaient pas. 


\section{(43)}

3) nos efforts vos intentions pour le bien de votre

) peuple. Tous les fardeaux qui lui furent in-

) posés n'ont eu qu'un motif, celui d'assurer votre

) trône et notre tranquillité : le jour où l'un et

") l'autre seront garantis, nous serons prêts à al-

” léger les charges qui pèsent sur lui. Mais rien

\# n'a encore été fait dans cet intérêt; la marche

》) incertaine de votre ministère n'a rien établi qui

) puisse servir à nous rassurer; au lieu de com-

”) primer l'esprit de révolution, c'est sous leurs

3) auspices qui prend un nouvel et dangereux essor;

3) au lieu de trouver en lui des garanties, nous

” devons en chercher contre lui : comment lui

》f ferions-nous de nouvelles concessions? Formez

) une autre administration qui soit toute dans l'in-

》) térêt d'établir la monarchic et les institutions

》qui peuvent assurer votre trône, qui comprenne ) le gouvernement que vous avez ćtabli par la ) Charte constitutionnelle, qui soit appuyée par

) tout ce qu'il y a de monarchique dans vos peu-

》) ples; et alors, rassurés sur sa marche par ses

) principes, toutes nos conditions seront faciles,

) parce que toutes nos concessions seront raison-

) nables; dans l'état actuel, elles ne le seraient pas.

ग) Tels sont les moyens les plus efficaces de con-

》) solider l'ordre intérieur en France, et d'asso-

) cier votre royaume au système de bonne intel-

») ligence et de pacification générale. »

Qui peut douter qu'un pareil langage ne portât dans l'esprit du Roi toute la conviction qui lui est nécessaire pour saisir et embrasser un système plus éclairé et plus sûr? La force de la raison et de la vérité dissiperait toutes les ombres que des passions intéressées entretiennent autour de lui. L'esprit du Roi est mieux préparé que celui d'aucun 


\section{(44)}

autre à envisager la lumière sans que son éclat blesse ses yeux. Qui peut mieux comprendre que lui que l'Europe ne peut mieux se préserver de la révolution renaissante en France, qu'en l'étouffant dans le sein de la France qui la recèle! que tous les moyens de s'en préserver au dehors, quand elle s'appuyerait de toutes les forces de ce pays, seraient inutiles et impossibles; que le premier devoir des alliés est de se garantir de cette terrible invasion; qu'ils ont le droit d'examiner par quels moyens on peut comprimer l'élan révolutionnaire qui se fait sentir en France; que ceux qui dans cet intérêt admettraient l'occupation militaire de la France, ou le partage de ses provinces, ou le changement de dynastie légitime, ébranleraient toutes les colonnes de l'édifice europ cén au lieu de le consolider; que le gouvernement représentatif que le Roi a donné à la France n'a été un obstacle à un établissement plus solide du trône, qu'en ce que les conditions n'en ont pas été comprises par ses ministres, et que leurs intérêts et leurs passions se sont opposés à son exécution franche et entière; que, bien connu, bien compris et franchement dirigé, on y eût trouvé toute la force de ses institutions; que ces intérêts et ces passions des ministres ne leur laissent plus la liberté de se rattacher aux doctrines et aux homnies de la monarchie; que la seule ancre de salut, au milieu de l'orage imminent, est de se rattacher à tout prix aux principes, aux institutions et aux hommes monarchiques; et qu'on ne peut y parvenir qu'en confiant le gouvernail aux hommes qui imposent une grande confiance et un grand assentiment à tous ceux qui veulent sauver le vaisseau de l'Etat? 


\section{(45)}

C'est dans une semblable détermination qùe peut se trouver notre dernière espérance; il serait bien malheureux pour nous, pour l'Europe entière, pour les générations à venir, qu'elle se perdît avec toutes les autres.

FI.N. 





\section{DIALOGUE}

E N TR E

\section{UN GRENADIER}

DE LA GARDE ROYALE

ET UN INGONNU. 



\section{DLAOGUE}

EYTR T:

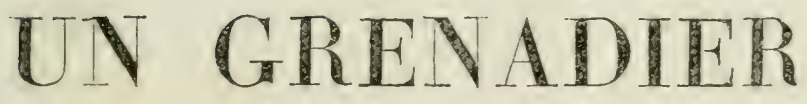

DE LA GARDE ROYALE

\section{ET UN INCONNU,}

REGUEILLI

PAR LE GAPITAINE DE CE GRENADIER.

$$
\text { PRIX : } 50 \text { CENTIMES. }
$$

\section{A PARIS,}

CHEZ DELAUYAY ET DENTU, LIBRAIRES,

$$
\text { PALAIS-ROYAL, GALERIE DE BOIS. }
$$$$
\text { I } 822 .
$$ 



\title{
DIALOGUE
}

E N T R E

\section{UN GRENADIER}

\author{
DE LA GARDE ROYALE
}

\section{E'T UN INGONNU.}

$\mathrm{U}_{\mathrm{N} \text { grenadier de ma compagnie, que je distingue }}$ depuis long-temps par son excellente conduite et son bon esprit, m'a rendu compte d'un entretien qui m'a paru curieux, et dans lequel il a fait briller un bon sens et une rectitude de jugement qui ne m'étonnent pas, depuis que je connais à fond ce brave homme. Cependant, quelque justesse d'idée qu'ait fait briller ce loyal soldat, privé d'éducation, dans le dialogue dont il m'a rendu compte, il faut observer qu'il n'est pas le seul capable de répondre ainsi, et je crois ètre sûr qu'il en est plus d'un qui, sondé de la même manière, a répondu dans le mème esprit à son insidieux interlociteur.

Ce militaire profitait d'une permission pour se rendre, de sa garnison aux environs de Paris, dans la capitale. La beauté du jour, le bon état 


\section{(6)}

des chemins, et son anciome habitude d'aller à pied d'un bout de l'Europe à l'autre, tout cela l'avait iuvité a faire pédestrement son petit voyage. Cheminant donc à son pas de route ordinaire, il se retoume et voit arriver derrierc lui un voyageur suivant la mème direction, $\mathrm{n}$ ais qui semBhat aroir grande hâte d'arriver, puisqu'il allongeait sensibiement le pas. Marchant ainsi, notre homme rejoint bientòt le soldat; mais alors il ne marche plus que l'allure de ce demier. Il faut noter que le voyageur était bien couvert, et qu'il n'avait mullcment l'air d'ètre piéton par nécessité. Ia conversation ne larda pas à s'engager. Voici comment :

Le Voyageur.-Bon jour, mon brave; vous arez l'air un peu fatigué. Il parail que c'est depuis long-lemps que vous faites le métier de fantassin?

Le Soldat. - Cuand on marche depuis vingtsept ans on n'est plus si léger; mais j'irais peulètre encore plus loin que bien d'autres, qui ne sont lestes que le premier jour.

Le $V^{2}$ - J'ai servi aussi, moi, et je suis content quand je rencontre un vieux troupier (soldat). J'espere que nous prendrons un verre de vin en arrivant à la barrière.

Ie S. - Je ne refuse jamais cela, quand je 
srois que c'est un brave homme qui me l'offie.

Le $V$. - Eh bien! à la bonne heure! c'est comme çà que j’aime que soit le soldat. Quand je vous ai vu de loin, j’étais sûr que vous étiez un de nos vieux, un des bons, et j'aurais mieux aimé courir que de ne pas vous rejoindre.

Le S.-Moi, je n'en dis pas autant; car si vous aviez été devant moi, il aurait bien fallu vous laisser aller.

Le $T$. - Depuis vingt-sept ans, vous devez en avoir joliment vu?

Le S. - Je vous en fais mon billet; et je sonhaitcrais plutòt à un de mes amis d'ètre aveugle. que d'en voir de cruelles comme moi.

Le $T$. - Est-ce que vous avez fait la campagne de Russie?

Le S. - Oni, monsieur; pour me remettre d'aroir un peu trop souvent tiré au mur (manqué de virres), et sué à l'armée de Portugal, jai réé me ratraichir dans la Russic-Blanche.

L. - Avez-rous été jusqu' ir Moscou?

L.e \$__ jai été jusqu’a Moscou! ah! je le crois bien: mais j'ai encore mieux fait, jen suis revenu, quoiqu'on entendit plus sourent le bruit du canon que le roulement de la soupe dans ce pays de misère.

Le $V$. - Cette malheurense sampanne nous a coûté bien cher. 
Le S. - Si on en avait fait dix comme celle-là, les vieux soldats seraient un peu plus rares en France que les hommes de cent ans.

Le $V$. - Je crois que vous avez raison. Dans quel corps serviez-vous alors?

Le S. - J'étais dans les grenadiers à pied de l'ex-Garde.

Le V.-Dans ce cas, vous deviez avoir des douceurs que les autres n'avaient pas, parce que l'Empereur avait soin de sa Garde.

Le S. - Oui, il en avait soin quand il y avait mèche (moyeu); mais quand il n'y avait que des sapins et de la neige, il ne pourait pas fabriquer des rations avec çà.

Le $V$. - Mais quand on trouvait des magasins, on songeait d'abord à vous.

Le S. - C'est comme vous dites, quand on trouvait des magasins; mais comme on ne trourait que des magasins de cendre, il fallait se serrer le ventre et laisser pousser ses dents les trois quarts du temps. On n'a battn que deux fois la berloque (hatteric pour la distribution des vivres) depuis Moscou; une fois à Smolensk, pour recevoir une ration de pain, une poignée de riz et une galette de biscuil; et une autre fois à Orscha, pour une ration de pain qu'on nous y a encore cionné. Aprés cela, il n'y a plus eu que 
distribution de draps blancs pour tout le monde. sur lesquels on a couché tous les jours jusqu'à Wilna.

Le $V$. - A Wilna, vous avez trouvé des ressources.

Le S. - Oui, avec leur argent; ceux qui en avaient, ont trouvé des barbes sales (des juifs) qui leur ont vendu du schnaps (eau-de-vie de pomme de terre) et du pain; mais pour les rizpain-sels (employés de l'armée) ils ne songeaient qu'à prendre leur clique et leur claque pour n'ètre pas cosaqués (pris par les Cosaques); il fallait voir comme ils filaient. Aussi les Russes ont dû trouver de quoi frire un peu mieux que nous, quand nous avons en délogé.

Le $V^{-}$. - Enfin, vous en êtes revenu, et je suis sûr que vous ètes toujours solide et bon là (bon soldat).

Le S. - Ah! pour çà; sill ne faut pas trop courir, et qu'il ne s'agisse que de jouer des fourchettes (marcher), porter le ridicule (le sac), et faire de la musique avec la clarinette de cinq pieds (le fusil), je peux bien encore compter pour un.

Le $V$. - Allez, on les compte, les braves comme vous, et j'espère bien qu'on les trouvera quand on aura besoin d'eux. N'est-ce pas qu'on peut compter sur vous? 
I.eS. - Je ne serais pas digne d'ètre soldat français, si on ne pouvait pas compter sur moi.

Le F. - Clect très-bien, l'ancien. D’après ce que vous máarez dit, vous n'ètes entié daus la Garde imperiale que pour faire la campagne de Russie : quand en ètes-vous sorti?

L. S.- Apus Jabdication de Fontainebleau. te me tromvais la, et je fus a l'ile d'Elbe; puis en 1315 , arres le licencioment de l'armée, j’entrai dans la Garde royale.

I.e $V .-A h ! \cdot m o n$ brave, vous avez été à l'île d'Elbe: vous des un de ces fideles à Napoléon que l'Europe admire.

Le S. - Je ne sais pas si ètre fidele est une chose si rare qu'il faille l'admirer; mais il me semble, dans tous les cas, que ce n'est pas senlement à Napoléon qu'il fallait l'ètre, et que, quand on sert le lioi, il fant que ce soit fidèlement ou ne pas s'en mneler. (Ici le voyageur parut, d'après l'observation da judicieux soldat, exprimer quelque chose de moins bienveillant sur sa physionomie, et il continua ainsi):

Le $V$. - Je ne dis pas qu'il faille ne pas être ficlèle au Roi, mais il faut aussi songer à la patrie.

Le S. - Ist-ce que servir le Roi n'est pas ser. 
vil la patrie? Je ne sais pas si vous ètes marle: ch bien! ('n supposant que vous le soyez, si je vous jouais un mauvais tour, est-ce que je nt ferais pas tort a rotre famille? Comne le Roi. est le père de tous les Français...., je mientends. et rous me comprenez bien.

Le $Г$... Ah! oui, je vous comprends; mais... Le S. - Il n'y a ni mais, ni si, ni quoi. J’ai juré d'ètre fidèle au lioi, et je dois tenir mors serment. Il n'en faut mène pas tant avec moi. Quand un honnet: homme promet quelque chose, c'est tache d'huile, et il n'y a que la canaille arec qui les promesses ne sont qu'une colle qui ne prenil pas. Si, pour ètre un brave. il fallait jurcr, et farre son monde au nème, (tromper), je me croirais insulté quand on me donnerait c: non là; et tout homme qui ne dit pas comme moi n'est qu'un....

Le $\%$ - Arrètez! arrélez! et ne vous emiportez pas, puisque nous sommes d'accord. J'aime le Roi autant que rous, et je suis suir quavec lui la Charte sera toujours conservée.

Le S. - Vous me parlez-là d'une chose que je ne comprends pas beauconp. Mais, est-ce que par hasard rous roudriez dire qu'apres lui il est à craindre quil ne reste pas de prince pour nous rendre henreux? Est-ce qu'ils n'ont pa: toujours ité hons, et trop bous dans cetto fi- 
mille? comme ils n'ont jamais pêché que parlà, je suis tranquille; et qui me dit du mal de l'un!, m'en dit de l'autre: ainsi, monsieur, tenezvous pour averti, et ne venez pas me chatouiller les orcilles d'une manière qui ne convient pas à un soldat de la Garde, parce que je....

Le $T$. - Mais mon cher, vous vous fàchez, je crois ? c'est que vous m'entendez mal.

$L$ " $S$. - Si vous voulez que je vous entende mieux, expliquez-vous d'une autre façon.

Le $\Gamma^{T}$. - Vous voilà plus calme, à ce qu'il me parait: nous a!lons nous entendre. Vous avez été à l'île d'Elbe, avec vos braves camarades, qui ne voulaient pas abandonner leur général malheurenx : voila ce que jappelle une conduite digne des plus grands éloges, et....

Le S. - J'ai été à l'île d'Elbe parce que je n'avais pas encore servi le Roi, et qu'en suivant mes camarades, je ne croyais pas mal faire. Voilà tout.

Le $V$. - Les circonstances vous ont mis ensuite dans le cas de servir le Roi, et vous voulez le bien servir, je ne vous bläme pas: vous voyez bien que nous sommes d'accord.

Le S. - Ah ! vous ne me blâmez pas : pour peu que çà continue, nos chiens pourront peutètre finir par chasser ensemble (nous pourrons finir par être d'accoid.) 
Le V- - Mais, dites-moi, ceux qui ont été labas avec Napoléon, ne sont-ils pas plus mal vus que les autres dans vots'e Garde?

Le S. - Ce n'est pas plus ma Garde que la vôtre: C'est la Garde.

Le $V$. - Il faut peser ses paroles avec vous: eh bien! la Garde.

Le S. - Comme je n'ai étudié que chez M. Butord, je n'ai pas la parole en main comme vous; mais je comprends tout de même. Je vous dirai donc puisque vous voulez le savoir, que l'on regarde mal dans la Garde, et que l'on y fait peu de cas, qu'on y refuse mème de rengager ceux qui ont été à l'île d'Elbe... (Ici le visage du vovageur s'épanouit un instant, parce que le soldat arlecta malicieusement une suspension, après avoir donné une intonation convenable aux phrases qu'il renait de prononcer) quand ils ne veulent plus se comporter en soldat; mais quand un troupier veut se bien conduire, on ne lui demande ni d'où il vient, ni où il a servi.

Le $V$. - Cependant, il y en a qui se plaignent.

Le S. - Oui : eh bien! Quand vous rencontrerez un de ses camarades-là, dites-lui de ma part qu'on se plaint de lui, je ne vous dis que çà.

Le $V$. - D'après ce que vous me dites, il paraitrait que vous avez mieux été traité que 


\section{( 14 )}

la plupart de vos eamaradis, dans la Garde. Le S. - C'est ce qui vous trompe, monsieur; car on n'y a pas deux poids et deux mesures pour ceux qui y servent. La croix que je porte m'a été accordée à l'ile d'Elbe, et on m'a donné mon brevet dans la Garde. Comme tous les services y sont récompenstés, on n'a eu égard qu'aux vingt-un ans de grenade sans reproches, que j’avais, quand j’ai été décoré; et j'ai cela de commun avec tous ceux qui étaient dans le mème cas que moi.

Le V. - A la bonne hemre! cela me fait plaisir de vous voir content.

Le S. - Tous les autres le seraient comme moi, s'ils n'étaient pas trop difficiles à contenter.

Le $V$. - Il y en a beaucoup qui se plaignent de n'avoir pas d'avancement.

Le s. - Pour satisfaire ceux-là, il n'y a qu'à en mettre une centaine de mille de plus à l'ombre (en enterrer) tous les ans. Mais dans ce cas, il y en aurait plus de quatre de ceux qui voudraient çà, qui descendraient la garde les premiers (seraient tués), au lieu d'avancer. Souhaite la guerre qui voudra: puur moi qui en suis revenu, et qui sais ce que c'est, je ne demande que la paix.

Le $\%$ - Vous pouvez avoir raison pour vous, mais il ne faut pas donner tout a fait tort 


\section{$(15)$}

à ceux qui voudraient faire quelques campagnes, puisqu'il faut cela pour avancer, et savoir ce que c'est que la guerre.

Le S. - Si vous tronvez que ce soit une chose à desirer pour aroir un grade de plus, et contenter sa curiosité, que de voir extcrmincr quelques centaines de mille hommes, et brûler desvillages pour en entendre pleurer les habitans en ne leur laissant pas seulement des pommes de terre, je suis surpris que vous restiez ici; car il me semble d'avoir entendu dire qu'il y a des pays où çà va bien, et même si bien, que les hommes s'y mangent (I), après s'ètre crevé la paillasse (éventré). Si on ne vous mangeait pas, vous pourriez y faire de bomnes boufailles ( de bons repas), et vous avanceriez. Pour moi, jt n'ai pas envie de cet avancement-là.

Le $V$. - Mais on pourrait dire, à vous entendre, que vous n'aimez pas la guerre.

Le S. - On ne dirait que la vérité, et on en aurait menti, si on disait qu'elle me fait peur; je l'ai assez faite, pour le prouver. Eh! vous, monsieur, sans être trop curieux, avez-vous vu brûler beaucoup de poudre? nous nous serons

(1) Les journaux ont parlé d'un des chefs insurgés de la Nouvelle-Grenade, qui se vante d'avoir mangé de la chair humaine. 
pent-être trouvés ensemble à quelque affaire.

Le $V$. J J'étais à la bataille de Jemmapes.

Le S. - Hé bien! si vous n’avez plus entendu ronfler le brutal (le canon), depuis ce tempslà, il ne doit pas vous avoir rendu sourd.

Le $V$. - Je l'ai bien entendu quelquefois encore depuis, mais pas tout à fait d'aussi près que vous.

Le S. - Ah ! tiens, je devine: vous aimez bien à voir la guerre, mais cest quand les autres la font; quand c'est tout profit pour vous. Je parie que vous étiez dans les vivres.

Le $V$. - C'est peut-être moi qui ai souvent fait faire votre pain.

Le s. - C'est peut-ètre vous qui in'avez si souvent appris à tirer au mur, en m'en laissant manquer.

Le $T$. - Que voulez-vous, quand il n'y avait pas de farine, on ne pouvait pas vous faire du pain.

Le 5. - Vous árez raison, on ne fait pas de pain sans farine; mais vous conviendrez qu'il y aurait en plus souvent de la farine, sil n'y avait pas eu tant de fariniers qui n'en trouvaient que pour eux.

Le $T$. - On criait contre les administrations souvent sans savoir pourquoi, et au bout du 


\section{(i7)}

compte, elles rendaient de grands services, parce qu'il en faut.

Le S. - Ah! il ien faut: ce n'est pas si sùr que du vinaigre, çà. Ce qui me fait croire, à moi, qu'on pourrait s'en passer, c'est que je n'ai jamais mangé de pain plus blanc, ni une meilleure ration en Espagne, qu'une fois que, me trourant sous les ordres du général Gardanne, il consentit à le laisser faire aux paysans, pour qu'il ne leur en couiầt pas si cher, et que nous fussions mieux ( $\mathrm{I}$ ). Les riz-pain-sels, ne se seraient peut-ètre pas contentés de gagner le son.

Le $V$. - Je vous pardonne tout ce que vous direz là-dessus, parce que c'est comme dit le proverbe: ventre affamé n'a point d'oreilies. Comme on vous a fait entendre, quand vous pâtissiez, que les employés de l'armée en étaient cause, il est impossible de vous faire entendre raison là-dessus.

Le $S$. - Je n'aurais pas si bien cru cela, si ces camarades avaient eté aussi maigres que moi à l'armée; mais s'ils étaient maigres, ce n'était qu'en arrivant. Il fallait voir comme ils étaient bientòt remplumés de toutes les manières. Aussi

(1) Le fait est réel, et est arrivé à Fuente-Guinaldo: sur la frontière de Portugal, en $\mathrm{i} \mathrm{S}_{\mathrm{I}} \mathrm{I}$. 
ils ont le diable au corps ceux-là, pour quion fasse la guerre.

Le $V$. - Tenez, pour vous faire voir que j'aime la paix, je la fais avec vous, en ne parlant plus de cela. Le pain qu'on vous donne est-il bon?

LeS. - S'il est bon! Je le crois bien, eh puis! Ce qu'il y a de meilleur, c'est que le four ne tombe jamais ( quand les soldats manquent de pain, ils disent: le four est tombé), et qu'il y en a toujours sur la planche. Tant que j’en aurai comme celui-là, je serai content, parce que je me rappelle de m’ètre brossé le ventre faute d'en avoir.

Le $V$. - Il y en a qui en mangent peut-être plus qu'ils ne voudraient, quand ils ont droit à leur congé et qu'on ne le leur donne pas.

Le S. - On voil bien que vous croyez avoir dix ans de moins, monsieur, puisque vous parlez comme rài. Quand le Roi est revenu, je n’avais fait encore que quinze ans cle plus, et on ne songeait pas à me donner mon congé; je n'en avais mème pas envie. Il fallait pour l'obtenir avoir au moins une patte cassée, et c'est ce qui fait que je ne m'en souciais pas. $A$ présent, si on vous retient quinze jours de plus, c'est bien rare, et le plus souvent on ne vous fait pas attendre vingtquatre heures. 
Le $V$. - Mais, à votre compte, vous derriez avoir le vôtre.

Le S. - Je l'aurais eu, si je l'avais voulu; mais je me suis rengagé pour quatre ans. J'acheverai la trentaine, et puis j'essaierai d'en faire quatre autres; deux si je ne peux pas faire plus: que voulez-vous, je crains de perdre le nord en perdant de vue le drapeau du régiment, ct j’ai peur d'avoir le mal du pass quand cela arrivera.

Le $V$. - Vous êtes bien heureux d'avoir ces idées-là; car c'est un état bien ingrat que l'état militaire.

L.S, - Un ancien camarade, qui n'a pas voulu s'engager avec moi dans la Garde, me disail çà, un jour que je l'ai rencontré à la chasse aux chiffons.

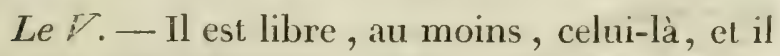
est son maître.

Le S. - C'est vrai, il a un f....u maitre, dont il dit qu'il est content; et il n'est pas obligé de se rendre à l'arpel tous les jours, mème pour dìner; mais, malgré çà, il y en a quelques-uns qui aiment micux ètre gènés comme moi, que libres comme lui. J'en connass un autre qui a fait la mème chose, et qui est venu l'autre jour avec une mine de carème et des habits d'ama- 


\section{( 20 )}

dou, tirer une carotte (faire une demande d'argent) à un de ses camarades, qui était sergent avec lui en 1815 , et qui est officier à présent dans le régiment. Il me semble que celui qui est officier a mieux fait que celui qui, pour être ce qu'il appelle libre, n'a pas voulu continuer ì servir.

Le $V$. - Qu'aurez-vous gagné après avoir servi vos trente ans?

Le S. - Ce que j'aurai gagné? l'hommeur d'avoir servi sans tàche, je crois, et puis l'avantage d'ètre un des meilleurs bourgcois de mon village de bois, si je ne meurs pas de chagrin quand je n'entendrai plus le tambour, et que je ne verrai plus que des pélins. Oh! C'est fini, il y a trop long-temps que je vois des cocardiers (des soldats), et j'aibien peur de ne pouvoir jamais leur dire adieu.

Le $V$. - Vous n'aurcz cependant pas un fàmeux traitement.

Le S. - Deux cent cinquante francs de ma légion, et au moins autant de retraite( $(1)$, vous croyez que je ne serai pas bien dans ma barraque.

(I) Voyez l'ordonnance du 20 février I 822 , entre autres les $n^{\text {os }} 34,43$ et 5 o. Ce sont de simples grenadiers ou voltigeurs de la Garde, qui ont 300 et 340 fr. de retraite. 


\section{(2I)}

Si je n'en étais pas sorti, je n'en aurais jamais tant gagné avec mes bras, et il aurait fallu partager avec ma femme, et cinq ou six dévorans qu'elle m'aurait pondus.

Le $V$. - Vos enfans, quand ils auraient été grands, vous auraient nourri.

Le $S^{\circ}$ - En connaissez-vous beaucoup qui soient bien nourris comme çà? Pour moi, je n'en connais point qui aient peur d'avoir des indigestions, et j'aime nieux avoir mon pain gagné que de l'attendre de mes enfans.

Le $V$. - Bien; d'après votre compte, à vous, vous aurez cinq cents francs.

Le S. - Je vous ai dit cinq cents francs au moins; mais si j'avais voulı, j'aurais cu un peu plus, je n'avais qu'à accepter les sardines (les galons de caporal), à présent je serais sergent et tant de gagné pour ma retraite, qui serait de cinq à six cent francs; mais je n'ai pas voulu de cet embarras-là.

Le $V$. - Commient, vous avez refusé de l'a vancement?

Le S. - Je le crois bien, quand on n'en sait pas plus que moi, il vaut mieux rester tranquille et faire son petit service d'officier de guérite (de soldat).

Le $V$. - Vous ne savez donc pas écrire. 


\section{(22)}

Le S. - J'écris bien un peu. Il est dommage seulement que je ne sache pas lire mon écriture le lendemain. Quand on n'est savant que comme çà, autant vaut ne savoir ni A ni B; aussi on ne m’a jamais demandé pour travailler chez le quartier-maitre:

Le $V$. - Vous en sauriez d'avantage, qu'une fois sergent vous en seriez resté là. Pour passer officier à présent, il faut avoir des lettres de noblesse.

Le S. - Qu'est-ce que c'est que des lettres de noblesse?

Le $V$. - Ce sont les titres qui prouvent que l'on est noble.

Le S. - $\Lambda$ h ... C'est çà : et les lettres de vigneron, valent-elles les lettres de noblesse?

Le $V$. - Elles devraient valoir mieux, car un vigneron est un homme utile.

Le S. - Je vous en réponds que c'est un homme utile. S'il n'y avait pas de ces braves gens-là, on ne boirait que de l'eau, et je ne chanterais jamais $l a$ mère Godichon; aussi on leur rend justice dans le Garde. Un sous-officier qui n'a pour noblesse que ses chevrons et dont le père ne fait qu'ume ou deux pièces de briolet (vins des environs de Paris) va passer officier dans le régiment. Que ne ferait-on pas pour le fils, si le papa faisait cent pièces de bon vin de Bourgogne ! 
Le $\boldsymbol{T}$. - Vous croyez qu'il passera; il n'est pas encore nommé. Cependant, par politique il est possible qu'on en fasse passer quelqu'un quin'est pas noble de temps en temps.

Le S. - Si c'est de la politique que l'avancement dans la Garde; elle est bonne pour les sousofficiers, yui n'ont pas, comme rous dites, des lettres de noblesse; car depuis que le régiment est formé, il y a eu des tours, et c'est toujours le leur qu'ön a suivi.'

Le $V$. - C'est fort bien : mais ces paurres vieux sous-officiers, quand ils sont parvenus à avoir l'épaulette, comment s'arrangent-ils avec lés autres officiers?

. Le S. - Comment ils s'arrangent? quoique je ne les fréquente pas, comme je ne les vois jamais faire deux bandes, je crois qu'ils vivent en camarades qui ne connaissent qu'un chemin, où ils sont sùrs de se trouver toujours ensemble, celui que le Roi hous montrera à tous quand il faudra marcher.

Le $V$. - Il parait que la composition des officiers supérieurs est un peu diffirente. Pour là, il n'y a pas moven d'y parvenir sans noblesse.

Le S. - C'est ce qui fait qu'un des derniers régimens de la Garde qui a changé de colonel a été donné à un brave soldat (je veux dire off- 


\section{( 24 )}

cier ) que j'ai vu blesser dix fois; et qui est noble comme moi.

Le $V$. - C'est étonnant cela! il ne doit y avoir que celui-là, qui n'ait pas ses titres.

Le $S$. - Des titres! Ils en ont tous à l'estime des braves gens; mais à la noblesse, il y en a eu depuis la formation de la Garde, et dans tous les grades, qui n'en avaient point. Ceux qui se plaignent d'être maltraités parce qu'ils ne sont pas nobles, seraient peut-être bien embarrassés pour dire quels services ils ont rendus ou voulu rendre au Roi. Ils n'avaient qu'à faire en sorte de ne pas se mettre dans cet embarras, et, nobles ou non, on aurait eu soin d'eux. Ne croyez-vous pas, monsieur?

Le $V$. Heu!... Heu!... je pense que tous les Français ne demandent qu’à bien servir.

Le S. - Tous; il me semble que vous faites tort à vos connaissances en disant cela. Si vous disiez beaucoup, à la bonne heure; mais tous, on a des preuves du contraire. Tenez, je crois qu'il n'y en a guère qui ne veuillent pas d'une place, et qu'il n'y en a que trop quelques-uns, dont le plus petit soucis est de la remplir fidèlement.

Le $V$. - Sait-on ce qu'il faut faire pour ètre fidèle?

LeS, - Si vous ne le savez pas encore, ce 


\section{$(25)$}

n'est pas moi qui irai vous l'apprendre; de plus habiles que moi y perdraient leur temps et leur latin, et ne vous l'apprendraient pas non plus.

Le $V$. Allons! Allons! Je vois que vous n'êles pas dans votre belle humeur aujourd'hui, et qu'il faut souvent changer de discours pour en trouver un qui vous plaise. Vous force-t-on d'aller à la messe le dimanche? le soldat français n'aime pas toutes ces cérémonies.

Le S. - Le soldat français qui n’a pas été élevé dans une basse-cour, a dù l'être dans une religion où il y a dus cérémonies plus ou moins, et il doit aimer à faire tout ce qu'on lui commande pour le service. Si j'étais catholique, il me semble que je n'attendrais pas qu'on me commandât pour aller à la messe, un jour d'obligation.

Le $V$. - Ah! vous n'ètes pas catholique. Comment faites-vous avec votre religion?

Le S. - Il y en a plus de quatre qui font comme mon chien avec la leur; moi, quand je suis dans un pays où il n'y a pas de prêche, je vais à la messe, parce qu'on ne m'a jamais dit qu'il y eût du mal de prier à ma manière, là où il y en a d'autres qui prient différemment. Quand je peux aller au prêche, j'en demande la permission, et on ne me le refuse que quand on ne peut pas faire autrement.

Le $V$. - Vous devez avoir des juifs aussi? 
Le S. - S'il y en a, personne ne les empêche d'aller à la synagogue.

Le $V$. - On a dù cependant vous tourmenter quand vous avez eu une mission, pour essayer de vous convertir.

Le S. - Comme on n'a forcé ni payé personne pour suivre la mission, il n'y a que ceux à qui çà a convenu qui se sont convertis, et il me semble que personne n'a rien à y dire, parce qu’ils en étaient bien les maî̀res. Tout de même un malin de la chambrée qui se moquait de ceux qui allaient à l'église, et qui se croyait un habile Grec parce qu'il avait oublié son Pater, a été pour se moquer aussi des missionnaires, et il s'est converti comme les autres.

Le $V$. - Etes - vous beaucoup de protestans dans votre régiment?

Le S. - Comme on n'a jamais dit à personne: es-tu protestant? es-tu juif? on n'en sait rien, ni moi non plus; et c'est comme m'a dit mon capitaine, une fois que je lui parlais de cela, si on voulait connaître combien il y en a, le mauvais monde ne manquerait pas de dire que c'est pour les mettre de còté; et qui sait si on n'en dirait pas davantage ( $\mathrm{I})$ ?

(I) Le Constitutionnel a fait, il y a environ deux ans, des articles à sa façon, dans lesquels il avertissait 


\section{(27)}

Le I._- Oh! il ne faut pas croire cela. Mais, dites-moi, les vieux soldats sont-ils contens de voir des étrangers dans la Garde?

Le S. - Comme ce ne sont pas les premiers qu'ils aient vus, et qu'il n'y a que les conscrits qui ne puissent pas se rappeler d'avoir vu des Polonais, qui élaient de nouvelles connaissances, ils n'ont pas eu besoin d'un grand effort pour s'accoutumer aux Suisses, qui sont d'anciens amis.

La $V$. - Ces braves Polonais! ils s'étaient si bien battus pour nous!

Le S. - Ah çà , c'est vrai; et il faut dire qu'il $\mathrm{y}$ avait de fameux lapins là dedans. Mais les voisins ne sont pas de mauvais camarades non plus; je les ai vus se taper solidement devant moi, tanłlis que j’étais en réserve à la Bérésina, et s'ils avaient fait demi-tour, il y serait bien resté quelques Français de plus, moi le premier, peut-être; et puis, ils ne se sont pas tapés que là.

charitablement les disciples de Calvin de prendre garde à eux, à cause de certain dénombrement ordonné, disait-il, par le Gouvernement. Eh! qu'on n'admire pas, après cela, la bonne foi et la prévoyance de ce conciliant et judicieux journal! 


\section{$(28)$}

Le V. - Ah! vous éticz en réserve à la Bérésina.

Le S. - Oui, pour que ce ne fût pas toujours la même chose; car nous étions là quelquesums qui avions été quelquefois à l'avant-garde, pour changer ( $\mathrm{I}$ ).

Le $V$. Je vois que vous êtes content, et je vous en fais mon compliment; mais, quoique vous en disiez, il y a bien de la différence d'autrefois à à présent.

Le S._- Oui, la différence est grande. Autrefois, pour obtenir une permission de découcher, il fallait qu'il fit bien beau temps; à présent, on n'en n'obtient plus... c'est fini... quand on ne le mérite pas; mais, autrement, on vous en accorde tant que vous voulez, sans compter. Autrefois, je me rappelle d'avoir vu le colonel $^{* *}$ (2) traiter, comme on dit qu'on traite les

(I) On doit se rappeler que ce soldat était grenadier à pied de l'ex-Garde, corps dans la composition duquel. il n'entrait que d'anciens militaires.

(2) Ce colonel est très-connu par le libéralisme qu'il affiche. Il est présumable que, pour être d'accord avec le respect qu'il professe maintenant pour les droits de l'homme, il a renoncé à faire les fonctions de caporal pour appliquer la discipline allemande. 
nègres, de paurres grenadiers qui n'avaient point commis de crimes; et à présent on ne vous dit pas plus haut que votre nom. Autrefois, on vous faisait le décompte tous les trente-rleux du mois, et à présent vous touchez, fin un centime, ce qu'il vous revient tous les trois mois. Autrefois, il fallait aller chercher son congé an royaume des taupes, si vous n'aviez pas le bonheur de vous faire couper un abatis (bras on jambe); à présent, quand vous avez fait vos six ans, vous allez où vous voulez, arec un certificat de bonne conduite, si vous l'avez mérité. Autrefois... ici on arrivait à la barrière. Le bourgeois interrogateur ne laissa pas acherer, et prit congé de son compagnon de voyage, en l'assurant que des affaires pressées l'obligeaient de longer le boulevard extérieur pour se rendre promptement à un rendez-vous.

Le grenadier m'a assuré qu'il n'anrait pas accepté le verre de vin dont il avait été question en s'abordant, parce que son acceptation ne l'engageait nullement avec l'homme qu'il venait de quitter.

Cet individu, lui dis-je, vous a bien fait roir sa façon de penser, et si vous l'eussiez laissé faire, il vous en eût bieu dit davantage. Dans ce cas, qu'auriez-vous fait? "Mon capitaine, me »dit-il, je sais bien qu’à présent cela n'est plus 


\section{( 30 )}

" à craindre; mais quand on aurait dù me cou"damner à la salle de police, comme un ser" gent de la Garde qui en avait arrèté deux, qui » disaient du mal du Roi, et vive l'autre (I), " je lui aurais mis la main dessus, et je ne l'au- rais pas plus lâché qu'un chat ne lâche un rat. "Si je le revois, je vous le ferai connaittre. "

Il parait que cet individu n'a plus reparu dans les garnisons du régiment.

(I) Il est aisé de deviner à quelle époque cela est arrivé.

DE LIIPRIMERIE DE C. J. THOUVÉ, RUE NEUVE-SANT-AUGUSTIT, $x^{0} \cdot 7$. 







\section{MOYENS}

de remédier aux maux de la France, et de la rendre florissante par l'agriculture.

Par J. A. Brosson, propriétaire cultivateur.

C'est le nombre du peuple et l'abondance des alimexs qui font la vraie force et la vraie richesse d'une nation.

FÉสย์นัศ.

Rendre productifs les terreins incultes, augmenter considérablement la valeur de ceux d'un mé-. diocre rapport; réparer beaucoup de routes; inspirer à nos soldats l'amour du travail, les rendre robustes et capables de supporter les plus grandes fatigues, les mettre à l'abri de la corruption des villes, leur faire reprendre avec plaisir, vigueur et intelligence, hors du service, les occupations rurales, qui, en améliorant leur sort, les mettront à mêne de se marier et d'élever leurs enfans; présenter aux officiers des spéculations avantageuses; rendre la France inattaquable sur plusieurs points de son territoire : tels sont les avantages qu'embrasse le projet que je présente.

Une des principales causes de la disette de grains qu'éprouve la plus grande partie de l'Europe, c'est la quantité immense de bras que les dernières, guerres, et surtout le gouvernement despotique et meurtrier de Bonaparte, ont enlevée 


\section{( 2)}

àl'agriculture. En effet, la classe agricole a fourní le plus de victimes à l'ambition du despole $\left(^{\star}\right)$, tandis que, sous un Prince sage, éclairé, elle doit être la plus ménagẹe, la plus honorée, puisqu'elle nourrit toutes les autres. Je ne rappelle ici l'extravagance du conquérant, qui a si bien servi nos ennemis, qu'afin que, connaissant la cause de nos maus, nous cherchions les meilleurs moyens de les réparer. Ces moyens, aussi simples que certains, nous les trouverons dans la fertilisation de notre sol; les autres n'étant que factices, on ne doit les employer que comme auxiliaires et supplémentaires.

Il n'y a rien de mieux dit, de plus exact, en agriculture, que le passage suivant tiré d'un de nos meilleurs écrivains: " La terre produit à proporlion du nombre des bras quila cultivent; mieux cultivée, elle rend davantage; cette surabondance de productions donne de quoi la culliver mieux encore : plus on y met d'hommes et de bétail, plus elle fournit d'excédent à leur entretien. On ne sait où peut s'arrêter cette augmentation continuelle et réciproque de produit et de cultivateurs. Au contraire, les terreins négligés perdent leur fertilité : moins un pays produit d'hommes, moins il produit de denrées; c'est le défaut d'habitans qui l'empêche de nourrir le peu qu'il a;

(*) Les campagnes ont fourni aux conscriptions, en raison de leur peu de population, beaucoup plus d'hommes que les villes, oin les personnes aisées ont presque toujours trouvé, soił par argent, soit par protections, les moyens de s'y squstraitse. 


\section{(3)}

ê dans tonte contrće qui se dépeuple, on doit tôt ou tard mourir de faim. »)

Mais je m'abstiendrai de parler des terres en pleine culture, que jabandonne an peu de surveillance et d'activité de la plupart de leurs propriétaires, et à l'ignorance routinière des laboureurs, pour fixer l'attention du Gouvernement plus spécialement sur celles que nous appelons mauvais terreins: quelques-unes sont susceptibles de produire des grains et des prairies artificielles, et toutes de se couvrir des plus beaux arbres.

Ces mauvais terreins se divisent en crayeux ou calcaires, tels que les plaines de la Champagne; en sablonneux, tels que les landes de Bordeaux; en pierreux, qui se trouvent dans tous les départemens; et en marécageux. Je ne porterai point sur notre plan ces derniers, dont l'insalubrité pourrait altérer la santé de nos travailleurs, qui, étant les défenseurs du trône et de la patrie, doivent être robustes et bien portaus, pour marcher à l'ennemi chaque fois que le Gouvernement le réclamera.

Il est prouvé par l'expérience que les terreins calcaires, bien défoncés, conviennent à plusieurs de nos plus beaux arbres, et surtout au pin, au noyer, dont la croissance s'y fait rapidement $\left({ }^{\star}\right)$. Teut-on en faire des prairies artificielles? le sainfoin, ce fourrage par excellence pour les chevaux, y pousse très-bien et dure lung-temps.

${ }^{*}$ ) La variété connue sous le nom de noyer de la St,-Jeun, 21 "̉̇aut pas sujelte à geler, doit être préférée, 


\section{(4)}

Le terrein sablonneux est généralement le plus propre aux arbres verts: semés ou plantés avec soin, ils y poussent avec une vigueur étonnante.

Le terrein pierreux, dont la culture est la plus difficile et la plus dispendieuse, par la nécessité de le défoncer très-avant, et par la destruction des outils qu'il faut y employer, est propre à tonte sorte de productions. Lue châtaignier, le chêne, l'orme, le sycomore, l'érable plane, le merisier, l'arbre de Sainte-Lucie, le bouleau, le tremble, le mélèze, le poirier (1), y viennent très-bien; et les grains que l'on y sème dounent une récolte de première qualité.

Quelque picrreux que soit un terrein, il est facile de l'amener à ce degré de fertilité : tous nos agronomes le savent comme moi, et nous en avons continuellement la preuve sous les yeux. Il existe dans beaucoup de villages de pauvres familles qui, propriétaires de quelques petits coins de mauvaises terres, les défoncent, les fument de la boue des chemins, et parviennent à ériger en jardin des roches que couvraient à peine quelques pouces de terre végétale. Si les propriétaires riches suivaient cet exemple, ils amélioreraient lears propriétés rurales, ils augmenteraient leurs revenus, et en

(1) Voyez l'ouvrage de M. Desfontaines, membre de l'Institut, et Professeur de botanique du Jardin du Roi : Histoire des Arbres et Arbrisseaux qui peuvent être cultive's en pleine terre sur le sol de la France, avec des observations sur les terreins et les climals qui conviennent à chaque espèce, sur les usages auxquels on peut les faire servir, etc. 2 vol. in-80, à Paris, chez Brosson, rue Pierre-Sarrazin, no 9. 


\section{(5)}

occupant les indigens, ils les détourneraient de la mendicité, et peut-être du brigandage.

Mais, dansl'état actuel des choses, le Gouvernement seul peut et doit, pour multiplier ses propres ressources, donner l'impulsion à l'agriculture : ces moyens, je le répète, me paraissent aussi simples que certains.

Chacun de nos départemens contient plus ou moins de terres incultes. Que chaque légion départementale soit autorisée à former une caisse dont les fonds seraient fournis par les officiers qui ont de la fortune, au moyen d'actions dont les intérêts seraient payés à raison de cinq pour cent par an. La légion achèterait en son nom une poìtion quelconque de terres incultes parmi celles que désigneraitle ministre de la guerre ou celui de l'intérieur.

Il serait utile d'établir le dépôt de chaque légion dans la ville la plus voisine du terrein, et de faire dans celui-ci de simples constructions pour y déposer les outils de culture, loger les soldats chargés de les garder, et recevoir même les soldats convalescens, que l'air champêtre rétablirait plus promptement.

On occuperait ley soldats à défoncer le meilleur du terrein, et à y planter les principaux légumes, pour avoir sous la main et en abondance une partie des vivres nécessaires. Ils formeraient ensuite des pépinières d'arbres propres au terrein, que l'on planterait à mesure qu'il serait en état de les recevoir. C'est ainsi qu'en peu d'années, et presque sans frais, la France se gamirait de nouvelles fo- 


\section{(6)}

rêts, et ne craindrait pas đe long-temps la disette de bois dont on l'a si souvent menacée. En supposant que chaque homme ne cultivât par an qu'un demi-arpent, cinquante mille hommes rendraient chaque année à l'agriculture vingt-cinq mille arpens. Quand la portion de terre achetée par la légion aurait été mise en pleine valeur par les soldats eux-mêmes, le conseil chargé de présider aux opérations et tous les actionnaires pourraient la vendre pour en acquérir d'autres. Les intérêts des fonds étant payés, le bénéfice serait partagé entre tous les individus composant la légion; par conséquent les officiers, persounellement intéressés, surveilleraient les travaux avec zèle, et les soldats se prêteraient volontiers à des occupations tendantes à leur assurer, à leur sortie du service, une honnête existence.

Si telles étaient les occupations de nos troupes, les habitans de la campagne, chez qui naguère la conscription portait la désolation, non-seulement à cause des périls de la guerre, mais aussi à cause de cette extrême paresse et de ces débauches de tout genre auxquelles le soldat s'habitue dans les garnisons, les habitans de la campagne, dis-je, verraient avec plaisir leurs enfans embrasser l'ćtat militaire, persuadés qu'ils y trouveraient un moyen infaillible de se rendre laborieux. Souvent ils les engageraient eux-mêmes à s'cnrôler dans nos légions, et l'armée se recruterait sans peine autant que pourraient l'exiger les besoins de l'Ĺtat. Utiles, et par conséquent estimés en temps de paix, ces soldats ciloyens seraient, en temps de guerre, din- 


\section{(7)}

vincibles défcnscurs de leur patrie, d'inébranlables soutiens d'un trône protecteur de l'industrie. C'est alors que l'intérêt de l'armée ne ferait qu'un avec celui de la nation; c'est alors que l'armée frouverait un appui dans la nation; c'est alors aussi qu'elle sentirait plus que jamais l'importance des travaux auxquels elle se serait livréc p̧endant la paix. Car, par où l'ennemi pénètret-il ordinairement en France pour marcher sur la capitale? par les vastes plaines de la Champagne. Que nos troupes en défrichent les terres calcaires; qu'elles y pratiquent des tranchées; qu'elles y forment des pépinières; et ces plaines si funestes et si stériles présenteront par suitè des bois intpénétrables, et les mêmes moyens de défense que l'invincible Tendée.

Pour avoir une connaissance exacte des terres qui sont incultes, et dont beaucoup appartiennent aux communes, chaque préfet fournirait au Gouvernement un tableau de celles qui se trourent daus son départ ement, en désignant leur nature, les végétaux qui y poussent, leur distance des grandes routes, villes et hameaux. On choisirait de préférence les terreins situés dans le voisinage des grandes routes, dont on pourrait confier et payer lentretien aux troupes chargées du défi: chement des terres voisines. Les pierres retirées des champs répareraient les routes, et la terre des routes, qui forme un excellent engrais, transportée sur les champs, contribuerait à les fertiliser: dou!b]e avantage qui n'exigerait pas de grands frais.

Comment nos soldats ne se livreraicnt-ils point 


\section{( 8 )}

volontiers à un travail qui doit tourner à leur profit, eux que l'on a vus, non-seulement à Boulogne, mais même en Egypte, en Italie, en Espagne, où presque toujours ils manquaient de pain, se plaire à élever des chaumières, à y former de pelits jardins qu'ils étaient obligés d'abandonner peu de jours après? Presque tous fils d'artisans ou de laboureurs, chacun d'eux serait employé, autant que possible, au métier dont il aurait déjà fait l'apprentissage avant d'entrer au service, et par conséquent nos légions trouveraient en elles-mêmes toutes les ressources nécessaires. Partageant ainsi leur temps entre les travaux de la campagne et les exercices militaires, auxquels des jours seraient consacrés, nos braves sauraient, en présence de l'ennemi, manier également les armes pour attaquer ou se défen'dre, et les outils nécessaires pour se retrancher. Préservés de la corruption des villes, ils entretiendraient, ils accroîtraient leurs forces et leur courage. Les querelles, et les duels qui en sont presque toujours la suite, deviendraient beaucoup plus rares. $\mathbf{A}$ l'exemple des phalanges invincibles des Romains, ils iraient des travaux de la campagne à la victoire, et cle la victoire préparer aux champs de nouveaux iriomphes; ils y puiseraient un plus grand amour de la patrie. Comme l'a fort bien dit un philosophe ancien, qui fut peut-être le plus grand capitaine de son siècle (Xénophon), "Les gerbes donnent à ceux qui les font croître le courage de les défendre; elles sont, dans les champs, comme un prix au milieu des jeux pour le vainqueur. ») 


\section{(9)}

Il peut encore résulter de l'exécution de ce projet un avantage réel pour la police : elle est faite exactement dans les villes par la Garde nationale, qu'il est presque impossible d'organiser dans les hameaux. Par-tout où il y aurait des troupes, elles en tiendraient lieu, tout en se livrant à leurs travaux.

Je ne finirais point si je voulais exposer tout ce que ce plan peut présenter d'utile; c'est une branche d'économie politique dont les ressources sont inépuisables. Le grand Sully les connaissait parfaitement ; il répétait continuellement à Henri IV, que sa gloire consistait daus le bonheur des hommes, et le bonheur des hommes dans les travaux de l'agriculture.

Profitons de la nature de notre sol ; le succès dénontrera aux propriétaires tout lo parti qu'ils peuvent tirer de leurs terres, et par une noble émulation, les progrès de l'agriculture deviendront rapides, les moeurs s'épureront, les haines s'éteindront, l'amour de la patrie augmentera en raison de l'aisance dont le plus grand nombre des habitans jouira par son travail; et cette aisance, on ne manquera pas de l'attribuer aux soins paternels du Souverain, et à la sagesse de nos lois et de nos institutions.

\section{Exemples de terreins améliorés.}

" J'étais, il y a quelques années, dit Bernardin de Saint-Pierre (Etudes de la Nature), en Normandie, chez un gentilhomme aisé, qui fait va- 


\section{(10)}

loir lui-même un grand pâlurage situé à mi-côle; sur un assez mauvais fonds. Il me promena taut autour de son vaste enclos, jusqu'à un espace considérable qui n'était couvert que de mousses, de prêles et de chardons; on n'y voyait pas un brin de bonne herbe. A la vérité cé terrein était à-la-fois ferrugineux et marécageux; on l'avait coupé de plusieurs trancliées pour en faire écouler les eaux; mais c'était en vain, rien n'y pouvait croître. Immédiatement au-dessous, il y avait une suite de petiles métairies, dont le fond était couvert de gazon frais, planté de pommiers chargés de fruits, et entouré de grands aunes. Je demandai à son possesseur pourquoi des terreins si voisins étaient de rapports si différens. Ils sont de même nature, me dit-il; et il y avait autrefois, sur le lieu où nous sommes, de petites maisons semblables à celles que vous voyez là. J'en ai fait l'acquisition, mais à ma perte. Leur's habitans ayant du loisir et peu de terre à soigner, l'émoussaient, l'échardonnaient, le fumaient, l'herbe y venait. Voulaient-ils y planter, ils y creusaient des trous, ils en ôtaient les pierres, et ils les remplissaient de bonne terre, qu'ils allaient chercher au fond des fossés et le long des chemins: leurs arbres prenaient racines et prospéraient. ’)

Un curé nouvellement arrivé dans un village de la Cliampagne pouilleuse, près de Sézanne, dont les environs ne produisaient rien, après avoir cxaminé la nature du terrein, pensa qu'en le réchauffant il deviendrait fertile, et retirerait de la 


\section{(11)}

misère ses habitans. Il engagea ceux-ci à élever beaucoup de pigeons, pour en meltre la fiente dans leurs cliamps. Les paysans suivirent ce conseil, firent un commerce considérable de pigeons, achetèrent la fiente de ceux des environs, et ferlilisèrent en peu de temps leurs terres.

Un propriétaire à deux lieues de Riom en Anvergne, près du lieu de naissance du général Desaix, avait une pièce de terre de huit arpens dont un ne produisait presque rien, à cause de l'eau qui y séjournait une partie de l'hiver. Il y fit creuser un fossé croisé de quarante toises sur deux cents, et de quarante-deux pouces de profondeur; il le fit remplir de pierres jusqu'aux trois quarts, couvrir ces pierres de terre, et disperser le reste de la terre sur la surface du terrein : les frais se montèrent à $9^{6}$ francs. L'année suivante, il y yécolta trente sacs de blé de plus que dans les meilleures récoltes précédentes.

M. de Tracy, membre de l'institut et pair de France, a une propriété rurale dans le Nivernais, sur les bords de la Loire : une portion de cette terre était inculte en $179^{5}$, et de plus regardée comme n'étant susceptible d'aucune espèce de culture. On s'est avisé d'y planter de la vigne, qui a étonné par ses progrès; de sorte que ce terrein, qui était sans valeur, se vend aujourd'hui, tout planté, jusqu'à quatre mille francs l'arpent.

M. Roy, membre de la Chambre des Députés, a établi, dans sa terre d'Entrain, de superbes pépinicres en arbres de loule espèce, qui y pous- 
sent arec une vigueur étonuante. Ce terrein ferrugineux avait été condamné à la stérilité comme absolument ingrat, parce que les céréales et les prairies artificielles n'avaient jamais pu y venir après de simples labours.

M. Joly-de-Fleury est propriétaire d'une terre située à cinq lieues de Paris, sur la route de MontIhéri à Corbeil, où il y avait autrefois une étendue considérable de sable jaune ne produisant rien. Un régisseur, aussi actif qu'intelligent, y a fait pousser et prospérer des pépinières d'arbres de toule espèce, et des bois-taillis , essence de chîtaignier, de la plus belle venue.

En 1810, j'entrepris de faire valoir une ferme. située à six lieues de Paris : elle est de cent soixante-quinze arpens, et produisait alors trois mille trois cents francs. A près l'avoir fait cultiver à mes frais pendant cinq ans, et y avoir dépensé en améliorations tout le revenu, je viens de la louer six mille francs, bien persuadé que le fermier actuel y fera mieux ses affaires que le précédent, s'il a autant de moyens pécuniaires, s'il est aussi actif et économe que son prédécesseur. Une pièce de terre de vingt-cinq arpens était regardée par l'ancien fermier comme très-mauvaise, parce qu'elle ne produisait jamais plus de 5 à 4 septiers de blé par arpent. J'avais remarqué que, dans les temps pluvieux, l'eau séjournait sur la surface d'une partie de ce terrein, et que l'autre partie en était beaucoup trop abreuvée, faute d'égout. Mon premier soin fut.de hausser les parties basses, en y faisant faire des tranchées que je remplissais de. 
pierres et de bonnes terres par-dessus. Tous les bords élevés par la terre végétale que la charrue y avait entraînée depuis des siècles, furent enlevés et répandus sur toute la surface du terrein. Un fossé large et profond fut pratiqué le long de la pièce dans sa partie la plus basse : cela fait, je la fis labourer et fumer comme de coutume. Tous mes frais extraordinaires, comptés bien exactement, se montèrent à six cents francs. Le produit de la récolte en blé froment d'excellente qualité fut de dix septiers par arpent au lieu de quatre: j'eus par conséquent cent cinquante septiers de grains pour une somme de six cents livres. L'année suivante l'avoine abonda dans la même proportion, et le terrein se trouve amélioré pour un graud nombre d'années. J'en fis autant pour plusieurs autres pièces de terres, et mes frais ont été suivis du même résultat. Au premier labour, le charretier était muni d'un paquet de petites branches de saule qu'il fichait en terre à côté de chaque roche qu'il rencontrait; ensuite un ouvrier l'arrachait : et pour cette opération, telle pierre ne m'a pas coûté deux sous, qui avait peut-ètre brisé clepuis dix ans pour plus de cent francs de charrues.

Un terrein de vingt-cinq perches, le long des murs de la ferme, où il n'y avait pas trois pouces de terre végétale, sur un fond de pierrailles et de carrière de meulière, coûta deux cent quarante francs à défoncer à trois et quatre pieds de profondeur. Commej'en voulais faire un jardiu, la bonne pierre servit à faire les murs de clôture, et toute 
la mauvaise fut conduite dans le chemin le plus près, où l'on prit, en place des pierres, au retour de la même voiture, quatre cents tomberées de terre de route, qui firent un excellent fonds. Les arbres fruitiers $\mathrm{y}$ ont fait des inerveilles, et sont, presque tous les ans, chargés de fruits extraordinaires par leur grosseur et leur qualité. En 1815 , je vendis pour cent cinquante francs d'artichaux et pour cent francs de choux d'Yorck; le tout sorti de ce petit jardin, ma consommation prise, sans compter beaucoup d'autres légumes.

Je satisferai avec bien du plaisir ceux qui desireront se convaincre par leurs propres yeux de ce que j’avance.

Capilalistes spéculateurs, devenez acquéreurs de terres en manvais état, et il n'en manque pas en France; ne craignez point d'employer une parlie de vos capitaux à les améliorer, en les faisant défoncer, border d'arbres, épierrer, égoutter par des tranchées et des fossés, terroter, marner, sabler, plâtrer, suivant la nature de leur sol et les ressources que vous pourrez y trouver; parquer, fumer à champ couvert, ce qui vous sera facile par la quantité de bétail de toute espèce que vos moyens pécuniaires vous permeltront d'avoir. Pour peu que vous surveilliez vos opérations rurales, vous doublerez votre fortune en quelques années, pourvu cependant que vous ne bâtissiez 


\section{( 15 )}

pas de châteaux. L'air champêtre fortifiera votre santé, ou la rétablira promptement si elle est délabrée. Vos enfans, en se livrant aux plaisirs innocens de la campagne, prendront des notions d'agriculture, et s'attacheront à vos propriétés : dèslors vous pouvez être certains qu'ils ne dissiperont pas un jour les biens que vous leur laisserez. Ils se trouveront préservés des suites trop fréquentes et trop funestes de la mauvaise société, da désneuviement et du libertinage des villes, etc., etc.

De l'Imprimerie de FEUGUERAY, rue du Cloître St.-Benoit, $n^{\circ} 4$. 


$30 \mathrm{k}$ 